\title{
The best regimens for chemo-naïve incurable non-squamous non-small cell lung cancer with a programmed death-ligand 1, tumor proportion score 1-49\%: a network meta-analysis
}

\author{
Nobuhiko Fukuda $^{1} \wedge$, Nobuyuki Horita ${ }^{1}$, Seigo Katakura ${ }^{1}$, Ho Namkoong ${ }^{2}$, Ayami Kaneko ${ }^{1}$, Kouhei Somekawa ${ }^{1}$, \\ Youichi Tagami ${ }^{1}$, Keisuke Watanabe ${ }^{1}$, Yu Hara ${ }^{1}$, Nobuaki Kobayashi ${ }^{1}$, Takeshi Kaneko ${ }^{1}$ \\ ${ }^{1}$ Department of Pulmonology, Yokohama City University Graduate School of Medicine, Yokohama, Japan; ${ }^{2}$ Department of Infectious Diseases, Keio \\ University School of Medicine, Tokyo, Japan \\ Contributions: (I) Conception and design: N Fukuda, N Horita; (II) Administrative support: N Horita; (III) Provision of study materials or patients: \\ N Fukuda, S Katakura, N Horita; (IV) Collection and assembly of data: N Fukuda, S Katakura, N Horita; (V) Data analysis and interpretation: N \\ Fukuda, N Horita; (VI) Manuscript writing: All authors; (VII) Final approval of manuscript: All authors. \\ Correspondence to: Nobuyuki Horita. Department of Pulmonology, Yokohama City University Graduate School of Medicine, Fukuura 3-9 Kanazawa- \\ Ku, Yokohama, Kanagawa 236-0004, Japan. Email: horitano@yokohama-cu.ac.jp.
}

Background: Non-small cell lung cancer (NSCLC) is the leading cause of cancer-related death worldwide. There is a rank order of the efficacy and safety of treatment options, including immune checkpoint inhibitors (ICIs), bevacizumab (Bev), and cytotoxic drugs. When patients have low programmed death-ligand 1 (PD-L1) expression, there are multiple options for treatment. In this study, we focused on ICI regimens in patients with non-squamous NSCLC with low PD-L1 expression and no driver alterations and assessed the efficacy of the regimens using network meta-analysis.

Methods: Randomized trials for incurable chemo-naïve non-squamous NSCLC were collected through electronic searches. The data were independently extracted and cross-checked by two investigators. The primary outcome of this analysis was overall survival (OS). A frequentist weighted least-squares approach random-model network meta-analysis was applied.

Results: Sixty-eight eligible studies and 22,619 patients were identified. Using a platinum + thirdgeneration cytotoxic agent regimen (platinum regimen) as a reference, the platinum regimen + pembrolizumab $(\mathrm{Pemb})$ [hazard ratio $(\mathrm{HR})=0.55,95 \%$ confidence interval $(\mathrm{CI}): 0.34-0.89, \mathrm{P}=0.015]$ showed the best OS, followed by the platinum regimen + nivolumab (Niv) + ipilimumab (Ipi) (HR =0.61, 95\% CI: 0.44-0.84, $\mathrm{P}=0.003)$ with no heterogeneity $\left(\mathrm{I}^{2}=0 \%, \mathrm{P}=0.348\right)$.

Conclusions: The addition of Pemb or Niv/Ipi to platinum-based chemotherapy seems to be a good therapeutic option for non-squamous NSCLC with a PD-L1 tumor proportion score (TPS) of 1-49\%.

Keywords: Lung neoplasms; molecular targeted therapy; immune checkpoint inhibitors (ICIs); systematic review; pembrolizumab (Pemb)

Submitted May 18, 2021. Accepted for publication Aug 17, 2021.

doi: $10.21037 /$ tlcr-21-419

View this article at: https://dx.doi.org/10.21037/tlcr-21-419

^ ORCID: 0000-0002-8498-2915. 


\section{Introduction}

Non-small cell lung cancer (NSCLC) is one of the most common cancers and the leading cause of cancerrelated death worldwide (1). Platinum doublet and triplet chemotherapies have been the standard of care for patients with inoperable NSCLC without actionable driver mutations or translocations. The development of immune checkpoint inhibitors (ICIs) has offered enhanced therapeutic options for a variety of malignancies (2). ICIs are effective for multiple types of cancers because they treat malignant tumors by blocking checkpoint proteins but not directly attacking tumor cells. For example, programmed cell death protein 1 (PD-1) and programmed death-ligand 1 (PD-L1) inhibitors prevent tumor cells from inactivating the immune system (3). Currently, the first-line therapeutic option for chemo-naïve patients with stage IV PD-L1-high [tumor proportion score (TPS) 50\% or higher] NSCLC without driver alterations is singleagent pembrolizumab (Pemb) (4-7). Several regimens combining ICIs, bevacizumab (Bev), and cytotoxic drugs are recommended even for patients with low or no PD-L1 expression (4-7), since they greatly improve the objective response rate, progression-free survival (PFS), and overall survival (OS) of patients without substantially increasing the risk of adverse events compared to reference platinum regimens without ICIs. Although kinase inhibitors targeting epidermal growth factor receptor (EGFR), anaplastic lymphoma kinase (ALK), ROS proto-oncogene 1 (ROS1), and B-raf proto-oncogene (BRAF) are highly effective for patients with specific actionable mutations or translocations, such kinase inhibitors are not recommended for NSCLC patients without these mutations or translocations (8).

Few randomized clinical trials (RCTs) have directly compared ICI regimens because such a trial requires a large number of participants to reveal small differences in outcomes. However, understanding differences in efficacy and safety among ICI regimens is vital because they are crucial for treatment selection. A network meta-analysis is the best analytical technique for enabling indirect comparison among multiple regimens. Our previous network meta-analysis published in 2017 evaluated only non-ICI, non-kinase inhibitor regimens for chemo-naïve incurable NSCLC (9). The aim of the current study is to update the previous network meta-analysis, focusing on ICI regimens and low PD-L1 expression (TPS 1-49\%) in nonsquamous NSCLC cases without driver alterations. We present the following article in accordance with PRISMA
NMA reporting checklist (available at https://dx.doi. org/10.21037/tlcr-21-419) (10).

\section{Methods}

\section{Protocol registration and overview}

The protocol of this study followed the PRISMA extension statement for network meta-analysis (10), and the study was registered at the University Hospital Medical Information Network Center, Japan (11). There are amendments to information in the protocol (Appendix 1). Patient informed consent and institutional review board approval were not required for a systematic review.

\section{Study search}

The MEDLINE, Embase, Web of Science Core Collection, and Cochrane Central Register of Controlled Trials databases were searched to identify eligible articles on October 15, 2020. The formula used for MEDLINE is shown in Appendix 2. Conflicts during the study selection were resolved by discussion between the two reviewers (NF and $\mathrm{SK}$ ) or inquiry to a third reviewer (NH).

\section{Inclusion criteria: publication type and trial design}

Individually randomized controlled trials of incurable NSCLC written in English were collected. Studies focusing on patients with driver mutations or translocations were excluded. A conference abstract was allowed. Trials including random assignment to maintenance therapy, second-line treatment, or later-line treatments were excluded.

\section{Inclusion criteria: treatment}

Eligible treatments included first-line chemotherapy, including cytotoxic agents, molecular targeted therapies, and ICIs. Trials adding angiogenesis inhibitor to the platinum regimen were included. ICIs could be used alone or in combination with platinum regimens.

While comparability between cisplatin (CDDP) and carboplatin (CBDCA) in the treatment of NSCLC is controversial $(12,13)$, recent trials have allowed either CDDP or CBDCA based on the physician's choice $(14,15)$. Similarly, one of the important RCTs regarding Pemb, Keynote-047, included a "CBDCA and either paclitaxel 
(Ptx) or nanoparticle albumin-bound Ptx (nabPtx)" arm. Therefore, we decided to allow selective administration of CDDP/CBDCA and Ptx/nabPtx for our analysis. Nedaplatin was distinguished from CDDP and CBDCA.

Some recent trials that evaluated adding ICIs to platinum regimens allowed physicians to choose from several platinum regimens; however, a regimen-based network meta-analysis cannot incorporate such trials. Therefore, two models were constructed for analysis. One model, which was termed the "main model", did not distinguish each platinum + third generation cytotoxic agent regimen (platinum regimen). The other model, which was termed the "separate model", recognized each platinum regimen individually.

Kinase inhibitors were beyond the scope of the study because a regimen with these medications was not standard for patients without driver alterations (4-7).

RCTs examining perioperative chemotherapy and combined chemoradiotherapy were excluded from this study.

\section{Inclusion criteria: patients}

Patients with advanced, locally advanced, or recurrent non-squamous NSCLC were included. We accepted the disease stage that was mentioned in an article regardless of the historical revisions of the tumor-node-metastasis classification. Patients were not excluded if they had a medical history of radiotherapy or surgery. However, one study focusing on patients with poor performance status and the elderly was excluded. An RCT of large cell neuroendocrine carcinoma was excluded.

Patients whose PD-L1 protein expression as determined by the TPS was $50 \%$ or higher were excluded because current guidelines recommend different treatment options for those with a TPS $<50 \%$ and those with a TPS $\geq 50 \%$. Patients with a TPS $<1 \%$ were also excluded because the number of patients with a TPS $<1 \%$ influenced the results. If a subset of the study population fit our criteria, the subset data were analyzed. For example, when a study separately provided data of three populations with TPS scores of $0 \%$, $1-49 \%$, and $\geq 50 \%$, we collected the data of the population with a TPS $1-49 \%$.

If a study focused on patients with squamous NSCLC, driver alterations, or a TPS $\geq 50 \%$, the study was excluded. However, a study without criteria regarding the pathological subclassification of NSCLC, driver gene mutations, and TPS was acceptable; otherwise, most NSCLC studies would have been excluded.

\section{Quality assessment}

The Cochrane Risk of Bias tool was used for the quality assessment. This assessment tool included selection, performance, detection, attrition, reporting, and other bias (16).

\section{Outcomes}

The primary outcome of this analysis was OS, evaluated using hazard ratio (HRos). The secondary endpoints were the HR for PFS (HRpfs), the odds ratio of adverse events based on the Common Terminology Criteria for Adverse Events grade III or higher (ORae) (17), and the OR of treatment-related death. Disease progression was assessed in compliance with the Response Evaluation Criteria in Solid Tumors guidelines published in 2000 or its 2009 revision (18). Imaging evaluation performed by blinded independent central reviewing was preferred, if available. The first adverse event of grade III or higher was counted even if a patient experienced two or more adverse events.

\section{Data extraction}

Characteristics of eligible studies, including the first author name, publication year, sample size, and trial outcome, such as HRos and its $95 \%$ confidence interval (CI), were extracted by two reviewers (NF and SK). Consensus was reached by discussion between the two reviewers and arbitration from a third reviewer $(\mathrm{NH})$. Survival updates were included if available. Parmar's method was accepted for the survival data (19). If available, data from intention-totreat analyses were selected. The treatment arm was named based on the drug combination, regardless of the dose, route, or schedule. We obtained data from a subgroup by subtraction using a fixed-model meta-analysis formula. For example, subtracting data of the "PD-L1 1-49\%" subgroup from data of the "PD-L1 <50\%" subgroup yielded the data of the "PD-L $1<1 \%$ " subgroup. The data on adverse events and treatment-related death could be for patients with any PD-L1 TPS or pathology because these stratified data were rarely described.

\section{Statistical analyses}

The frequentist weighted least squares approach random- 

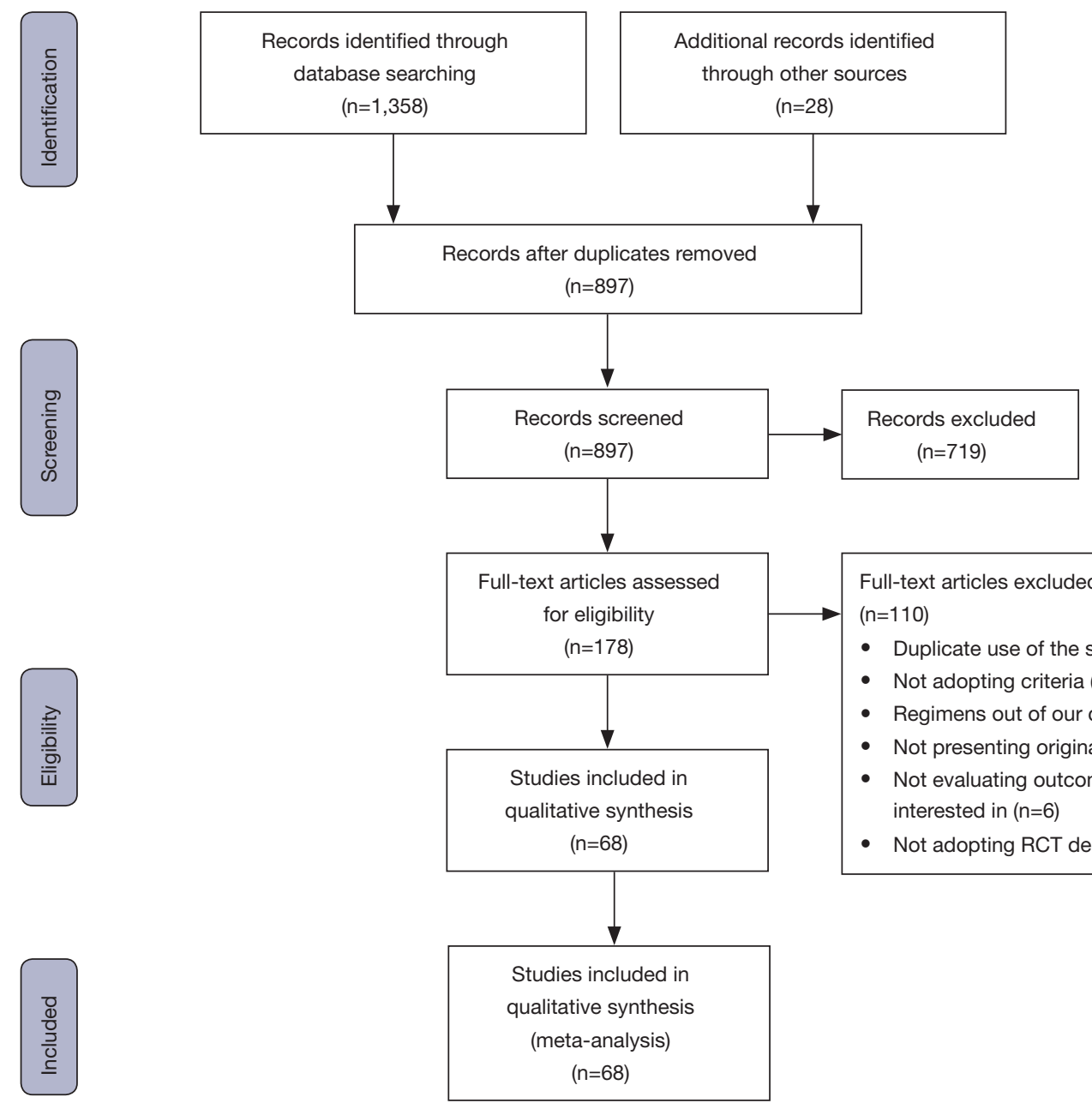

Full-text articles excluded, with reasons $(n=110)$

- Duplicate use of the same data $(n=50)$

- Not adopting criteria $(n=27)$

- Regimens out of our concern $(n=12)$

- Not presenting original data $(n=13)$

- Not evaluating outcomes that we were interested in $(n=6)$

- Not adopting RCT design $(n=2)$

Figure 1 Preferred Reporting Items for Systematic Reviews and Meta-Analyses flow chart for study search. RCT, randomized clinical trials.

model network meta-analysis was applied in our study (20). The OR of a binary outcome was continuously corrected with 0.5 , if necessary. The OR and HR were log-converted. In the separate model, platinum + pemetrexed (Pemt) was used as a common reference comparator because this regimen was selected for non-squamous carcinoma in recent trials $(14,15)$. Data were analyzed using $\mathrm{R}$ software (Command: netmeta, Package: netmeta) (21). The significance threshold was set at $\mathrm{P}<0.05$.

\section{Results}

\section{Study search}

We identified 1386 articles by electronic and manual searches. Of the 897 articles that met the preliminary criteria, 489, 719, and 110 were excluded through removal of duplicated studies, title/abstract screening, and full article review, respectively. We identified 68 eligible studies (Figure 1, Appendix 3).

\section{Characteristics of the included studies}

The main model had 26 studies and 53 arms, of which 16 included ICIs. The separate model had 63 studies and 130 arms, of which 9 included ICIs. The median and average age of patients ranged from 51 years to 67 years, with 50 studies having a median/average age in the $60 \mathrm{~s}$. The total number of patients was 22,619 , and the number of randomized patients in each study ranged from 41 to 1,252 , with a median of 248 (Table 1, Appendix 4).

According to the Cochrane Risk of Bias evaluation, all but one of the studies had at least one domain with a high risk of bias (Table $\mathrm{S} 1$ ). 
Table 1 Characteristics of included studies

\begin{tabular}{|c|c|c|c|c|c|c|c|c|c|c|}
\hline Study & Region & Phase & $\begin{array}{l}\text { Primary } \\
\text { outcome }\end{array}$ & Pathology & Arm & Stage & PS & Regimens & Pts & $\begin{array}{l}\text { Median } \\
\text { age }\end{array}$ \\
\hline Belani, 2017 & India & $\begin{array}{l}\text { Not } \\
\text { available }\end{array}$ & os & NSCLC & 2 & IIIb, IV & $\begin{array}{l}\text { ECOG } \\
0-1\end{array}$ & $\begin{array}{l}\operatorname{CDDP}\left(100 \mathrm{mg} / \mathrm{m}^{2}\right)+\mathrm{Ptx} \\
\left(175 \mathrm{mg} / \mathrm{m}^{2}\right)+\mathrm{CADI}-05 \\
(0.2 \mathrm{~mL}) \\
\operatorname{CDDP}\left(100 \mathrm{mg} / \mathrm{m}^{2}\right)+\mathrm{Ptx} \\
\left(175 \mathrm{mg} / \mathrm{m}^{2}\right)[\mathrm{M}][\mathrm{S}]\end{array}$ & 221 & 58 \\
\hline Carbone, 2017 & USA & III & PFS & NSCLC & 2 & IV, Rec & $\begin{array}{l}\text { ECOG } \\
0-1\end{array}$ & $\begin{array}{l}\text { Niv ( } 3 \mathrm{mg} / \mathrm{kg} \text {, q2w) } \\
1 \text { of } 5 \text { platinum } \\
\text { doublets [M] }\end{array}$ & 327 & 64 \\
\hline Chen, 2007 & Taiwan & II & $\mathrm{RR}$ & NSCLC & 2 & IIllb, IV & $\begin{array}{l}\text { ECOG } \\
0-2\end{array}$ & $\begin{array}{l}\operatorname{CDDP}\left(60 \mathrm{mg} / \mathrm{m}^{2}\right)+\mathrm{Vnr} \\
{\left[25 \mathrm{mg} / \mathrm{m}^{2}(\mathrm{~d} 1,8)\right]} \\
\operatorname{CDDP}\left(60 \mathrm{mg} / \mathrm{m}^{2}\right)+\mathrm{Dtx} \\
\left(60 \mathrm{mg} / \mathrm{m}^{2}\right)[\mathrm{S}]\end{array}$ & 94 & 63 \\
\hline Che, 2004 & Taiwan & II & $\begin{array}{l}\text { Not } \\
\text { available }\end{array}$ & NSCLC & 2 & IIllb, IV & $\begin{array}{l}\text { ECOG } \\
0-2\end{array}$ & $\begin{array}{l}\text { CDDP }\left[60 \mathrm{mg} / \mathrm{m}^{2}(\mathrm{~d} 15)\right]+ \\
\operatorname{Ptx}\left[66 \mathrm{mg} / \mathrm{m}^{2}(\mathrm{~d} 1,8,15)\right] \\
\text { CDDP }\left[60 \mathrm{mg} / \mathrm{m}^{2}(\mathrm{~d} 15)\right]+ \\
\operatorname{Vnr}\left[23 \mathrm{mg} / \mathrm{m}^{2}(\mathrm{~d} 1,8,15)\right] \\
{[\mathrm{S}]}\end{array}$ & 140 & 65 \\
\hline Doebele, 2015 & USA & II & PFS & NSq & 2 & IV & $\begin{array}{l}\text { ECOG } \\
0-2\end{array}$ & $\begin{array}{l}{\left[\text { CDDP }\left(75 \mathrm{mg} / \mathrm{m}^{2}\right) \text { or }\right.} \\
\text { CDBCA }(A \cup C 6)]+ \text { Pemt } \\
\left(500 \mathrm{mg} / \mathrm{m}^{2}\right)+\text { Ram } \\
(10 \mathrm{mg} / \mathrm{kg}) \\
{\left[\text { CDDP }\left(75 \mathrm{mg} / \mathrm{m}^{2}\right) \text { or }\right.} \\
\text { CDBCA }(A \cup C 6)]+ \text { Pemt } \\
\left(500 \mathrm{mg} / \mathrm{m}^{2}\right)[\mathrm{M}][\mathrm{S}]\end{array}$ & 140 & 64 \\
\hline Douillard, 2005 & France & II & $\mathrm{RR}$ & NSCLC & 2 & IV & $\begin{array}{l}\text { ECOG } \\
0-2\end{array}$ & $\begin{array}{l}\operatorname{CDDP}\left(100 \mathrm{mg} / \mathrm{m}^{2}\right)+\operatorname{Dtx} \\
\left(75 \mathrm{mg} / \mathrm{m}^{2}\right) \\
\operatorname{CDDP}\left(100 \mathrm{mg} / \mathrm{m}^{2}\right)+\mathrm{Vnr} \\
{\left[30 \mathrm{mg} / \mathrm{m}^{2}(\mathrm{~d} 1,8)\right][\mathrm{S}]}\end{array}$ & 239 & 57 \\
\hline
\end{tabular}

Table 1 (continued) 
Table 1 (continued)

\begin{tabular}{|c|c|c|c|c|c|c|c|c|c|c|}
\hline Study & Region & Phase & $\begin{array}{l}\text { Primary } \\
\text { outcome }\end{array}$ & Pathology & Arm & Stage & PS & Regimens & Pts & $\begin{array}{l}\text { Median } \\
\text { age }\end{array}$ \\
\hline Edelman, 2004 & USA & II & os & NSCLC & 2 & IIIb, IV & $\begin{array}{l}\text { ECOG } \\
0-1\end{array}$ & $\begin{array}{l}\text { CDBCA (AUC 5.5) + Gem } \\
{\left[1,000 \mathrm{mg} / \mathrm{m}^{2}(\mathrm{~d} 1,8)\right]} \\
\operatorname{CDDP}\left(100 \mathrm{mg} / \mathrm{m}^{2}\right)+\mathrm{Vnr} \\
{\left[25 \mathrm{mg} / \mathrm{m}^{2}(\mathrm{~d} 1,8)\right][\mathrm{S}]}\end{array}$ & 204 & 60 \\
\hline Galetta, 2015 & Italy & III & QOL & NSq & 2 & IIIb, IV & $\begin{array}{l}\text { ECOG } \\
0-1\end{array}$ & $\begin{array}{l}\operatorname{CDDP}\left(75 \mathrm{mg} / \mathrm{m}^{2}\right)+\text { Pemt } \\
\left(500 \mathrm{mg} / \mathrm{m}^{2}\right) \\
\text { CDBCA }(\text { AUC } 6)+\operatorname{Ptx} \\
\left(200 \mathrm{mg} / \mathrm{m}^{2}\right)+\mathrm{Bev} \\
(15 \mathrm{mg} / \mathrm{kg})[\mathrm{S}]\end{array}$ & 118 & 62 \\
\hline Gandhi, 2018 & USA & III & OS PFS & NSq & 2 & $\begin{array}{l}\text { III, IV, } \\
\text { Rec }\end{array}$ & $\begin{array}{l}\text { ECOG } \\
0-1\end{array}$ & $\begin{array}{l}{\left[\text { CDDP }\left(75 \mathrm{mg} / \mathrm{m}^{2}\right) \text { or }\right.} \\
\text { CDBCA }(A \cup C 5)]+ \text { Pemt } \\
\left(500 \mathrm{mg} / \mathrm{m}^{2}\right)+\text { Pemb } \\
(200 \mathrm{mg}) \\
{\left[\text { CDDP }\left(75 \mathrm{mg} / \mathrm{m}^{2}\right) \text { or }\right.} \\
\text { CDBCA }(A \cup C 5)]+ \text { Pemt } \\
\left(500 \mathrm{mg} / \mathrm{m}^{2}\right)[\mathrm{M}][\mathrm{S}]\end{array}$ & 186 & 64 \\
\hline Gebbia, 2010 & Italy & II & $\begin{array}{l}\text { QOL, } \\
\text { symptom, } \\
\mathrm{AE}\end{array}$ & NSCLC & 2 & IIIb, IV & $\begin{array}{l}\text { ECOG } \\
0-1\end{array}$ & $\begin{array}{l}\operatorname{CDDP}\left(75 \mathrm{mg} / \mathrm{m}^{2}\right)+\operatorname{Dtx} \\
\left(75 \mathrm{mg} / \mathrm{m}^{2}\right) \\
\operatorname{CDDP}\left(80 \mathrm{mg} / \mathrm{m}^{2}\right)+\mathrm{Vnr} \\
{\left[30 \mathrm{mg} / \mathrm{m}^{2}(\mathrm{~d} 1,8)\right][\mathrm{S}]}\end{array}$ & 86 & 62 \\
\hline Gebbia, 2003 & Italy & III & TTP \& OS & NSCLC & $2^{\ddagger}$ & IIIb, IV & $\begin{array}{l}\text { ECOG } \\
0-2\end{array}$ & $\begin{array}{l}\operatorname{CDDP}\left(100 \mathrm{mg} / \mathrm{m}^{2}\right)+\mathrm{Vnr} \\
{\left[25 \mathrm{mg} / \mathrm{m}^{2}(\mathrm{~d} 1,8)\right]} \\
\operatorname{CDDP}\left(100 \mathrm{mg} / \mathrm{m}^{2}\right)+ \\
\text { Gem }\left[1,400 \mathrm{mg} / \mathrm{m}^{2}\right. \\
\text { (d } 1,8)][\mathrm{S}]\end{array}$ & 278 & 62 \\
\hline
\end{tabular}

Table 1 (continued) 
Table 1 (continued)

\begin{tabular}{|c|c|c|c|c|c|c|c|c|c|c|}
\hline Study & Region & Phase & $\begin{array}{l}\text { Primary } \\
\text { outcome }\end{array}$ & Pathology & Arm & Stage & PS & Regimens & Pts & $\begin{array}{l}\text { Median } \\
\text { age }\end{array}$ \\
\hline Gronberg, 2009 & Norway & III & QOL & NSCLC & 2 & IIIb, IV & $\begin{array}{l}\text { ECOG } \\
0-2\end{array}$ & 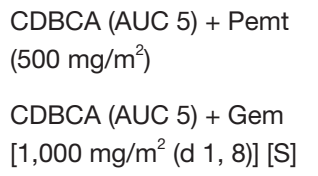 & 329 & 65 \\
\hline Hellmann, 2019 & USA & III & OS & NSCLC & $2^{\dagger}$ & IV, Rec & $\begin{array}{l}\text { ECOG } \\
0-1\end{array}$ & $\begin{array}{l}\text { Niv }(240 \mathrm{mg} / \mathrm{kg} \text { q2w or } \\
360 \mathrm{mg} / \mathrm{kg} \mathrm{q} 3 \mathrm{w})+ \text { Ipi } \\
(1 \mathrm{mg} / \mathrm{kg} \mathrm{q6w}) \\
{\left[\text { CDDP }\left(75 \mathrm{mg} / \mathrm{m}^{2}\right) \text { or }\right.} \\
\text { CDBCA }(\mathrm{AUC} 5)]+ \text { Gem } \\
\left(1,000 \mathrm{mg} / \mathrm{m}^{2}\right) / \mathrm{Pemt} \\
\left(500 \mathrm{mg} / \mathrm{m}^{2}\right)[\mathrm{M}][\mathrm{S}]\end{array}$ & 396 & 63 \\
\hline Herbst, 2020 & USA & III & os & NSCLC & 2 & IV & $\begin{array}{l}\text { ECOG } \\
0-1\end{array}$ & $\begin{array}{l}\text { Atz }(1,200 \mathrm{mg}) \\
{\left[\text { CDDP }\left(75 \mathrm{mg} / \mathrm{m}^{2}\right) \text { or }\right.} \\
\text { CDBCA }(A \cup C 6)]+ \text { Pemt } \\
500 \mathrm{mg} / \mathrm{m}^{2}[\mathrm{M}]\end{array}$ & 572 & 65 \\
\hline Kaira, 2019 & Japan & II & PFS & NSq & 2 & $\begin{array}{l}\text { III, IV, } \\
\text { Rec }\end{array}$ & $\begin{array}{l}\text { ECOG } \\
0-1\end{array}$ & $\begin{array}{l}\operatorname{CDDP}\left(60 \mathrm{mg} / \mathrm{m}^{2}\right)+\mathrm{S} 1 \\
\left(80 \mathrm{mg} / \mathrm{m}^{2}\right)+\mathrm{Bev} \\
(15 \mathrm{mg} / \mathrm{kg}) \\
\operatorname{CDDP}\left(60 \mathrm{mg} / \mathrm{m}^{2}\right)+\text { Pemt } \\
\left(500 \mathrm{mg} / \mathrm{m}^{2}\right)+\mathrm{Bev} \\
(15 \mathrm{mg} / \mathrm{kg})[\mathrm{S}]\end{array}$ & 48 & 65 \\
\hline Kawahara, 2013 & Japan & II & PFS & NSCLC & 2 & $\begin{array}{l}\text { Illb, IV, } \\
\text { Rec }\end{array}$ & $\begin{array}{l}\text { ECOG } \\
0-1\end{array}$ & $\begin{array}{l}\text { CDBCA (AUC 6) + Dtx } \\
\left(60 \mathrm{mg} / \mathrm{m}^{2}\right) \\
\text { CDBCA (AUC 6) + Ptx } \\
\left(200 \mathrm{mg} / \mathrm{m}^{2}\right)[\mathrm{S}]\end{array}$ & 90 & 67 \\
\hline Khodadad, 2014 & Iran & $\begin{array}{l}\text { Not } \\
\text { available }\end{array}$ & PFS & NSCLC & 2 & IIIb, IV & $\begin{array}{l}\text { ECOG } \\
0-2\end{array}$ & $\begin{array}{l}\operatorname{CDDP}\left(75 \mathrm{mg} / \mathrm{m}^{2}\right)+\mathrm{Dtx} \\
\left(75 \mathrm{mg} / \mathrm{m}^{2}\right) \\
\operatorname{CDBCA}(\mathrm{AUC} 5)+\operatorname{Ptx} \\
\left(200 \mathrm{mg} / \mathrm{m}^{2}\right)[\mathrm{S}]\end{array}$ & 100 & 51 \\
\hline
\end{tabular}

Table 1 (continued) 
Table 1 (continued)

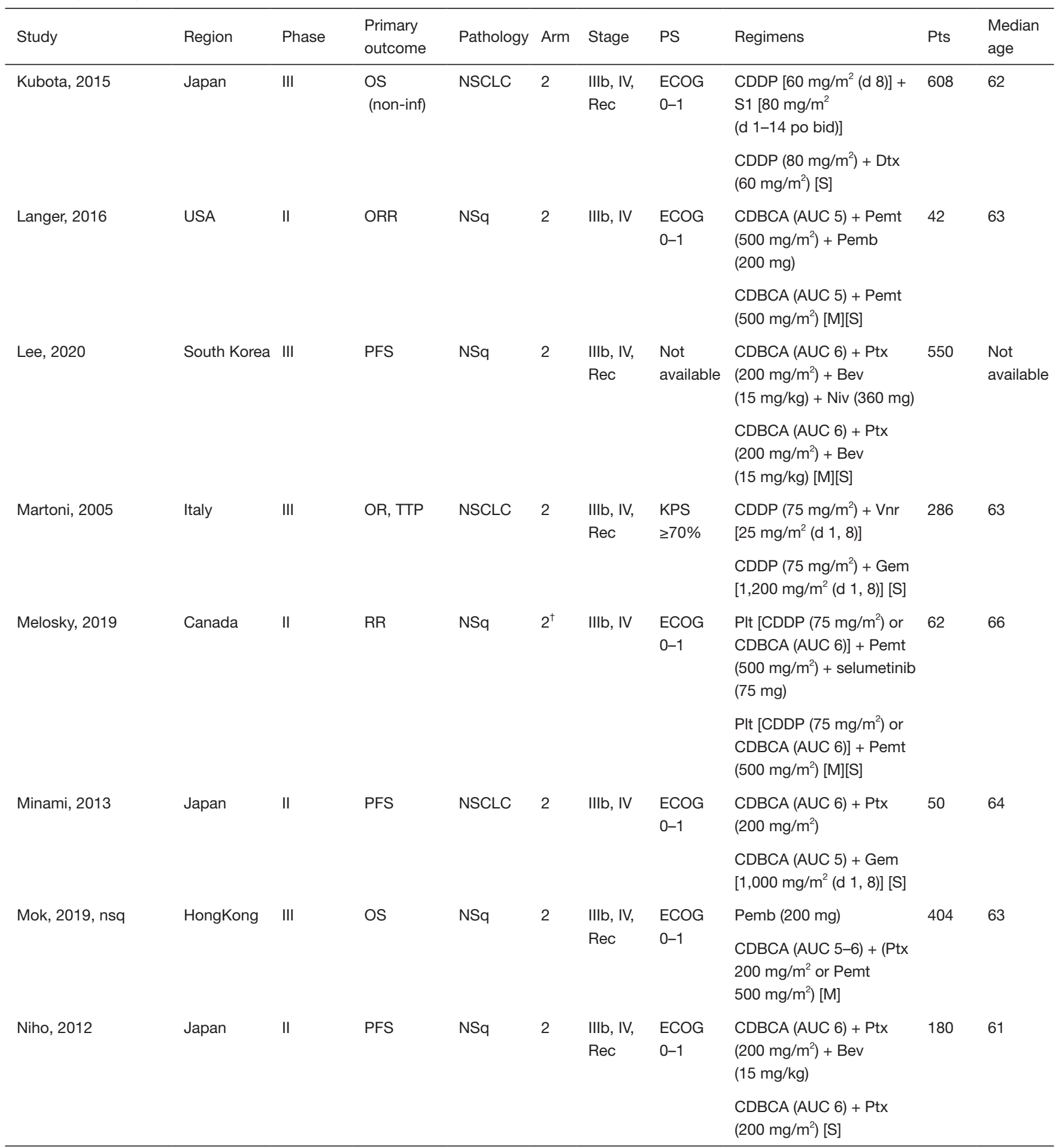

Table 1 (continued) 
Table 1 (continued)

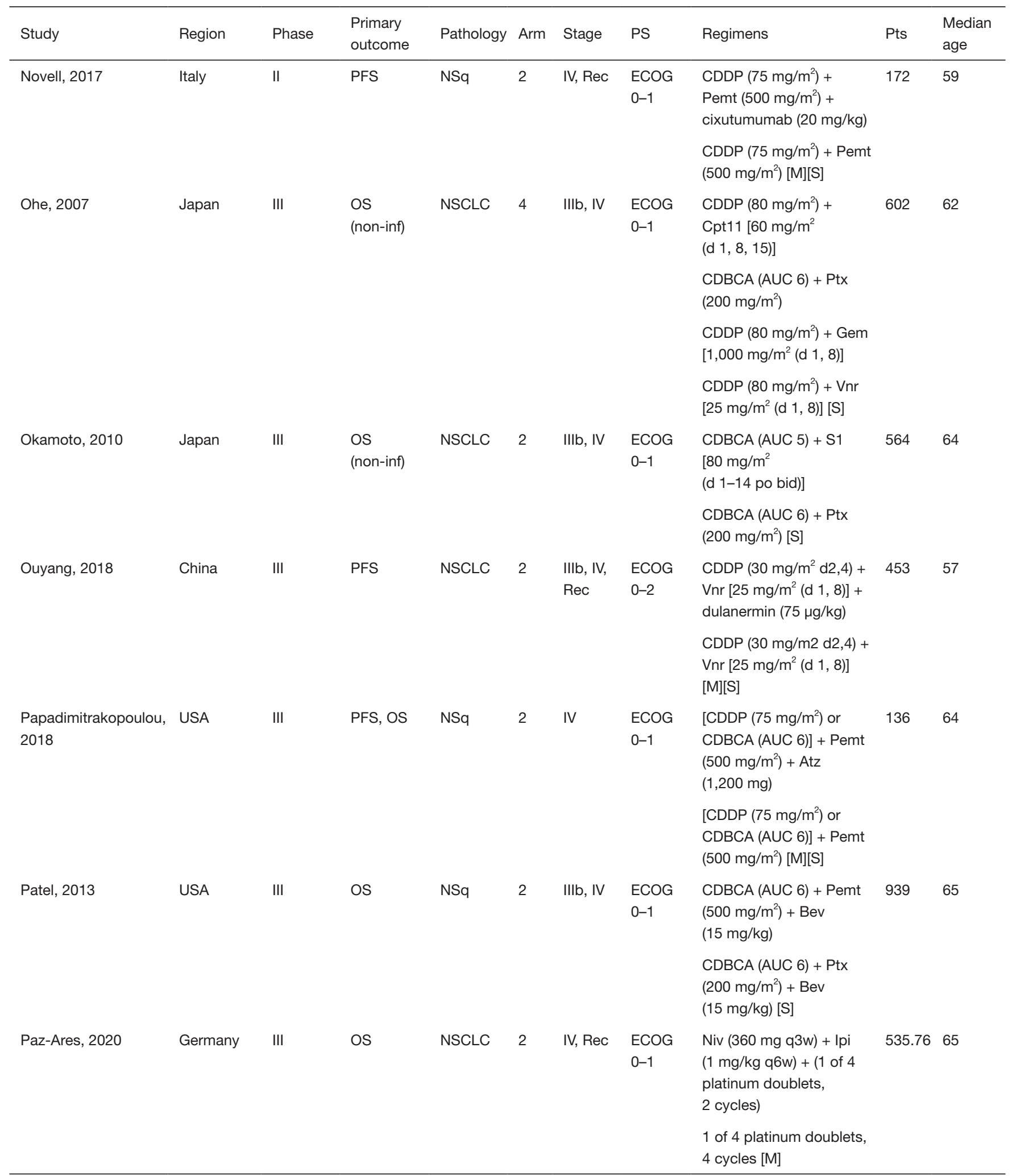

Table 1 (continued) 
Table 1 (continued)

\begin{tabular}{|c|c|c|c|c|c|c|c|c|c|c|}
\hline Study & Region & Phase & $\begin{array}{l}\text { Primary } \\
\text { outcome }\end{array}$ & Pathology & Arm & Stage & PS & Regimens & Pts & $\begin{array}{l}\text { Median } \\
\text { age }\end{array}$ \\
\hline $\begin{array}{l}\text { Ramalingam, 2017, } \\
\text { NSq }\end{array}$ & USA & II & PFS & NSq & 2 & IV & $\begin{array}{l}\text { ECOG } \\
0-1\end{array}$ & $\begin{array}{l}\text { CDBCA }(\text { AUC 6) + Ptx } \\
\left(200 \mathrm{mg} / \mathrm{m}^{2}\right)+\text { veliparib } \\
(120 \mathrm{mg}) \\
\text { CDBCA }(\text { AUC 6) + Ptx } \\
\left(200 \mathrm{mg} / \mathrm{m}^{2}\right)[\mathrm{M}][\mathrm{S}]\end{array}$ & 82 & 63 \\
\hline Rodrigues, 2011 & Argentina & III & G3/4PFS & NSq & 2 & IIIb, IV & $\begin{array}{l}\text { ECOG } \\
0-2\end{array}$ & $\begin{array}{l}\text { CDBCA }(A \cup C 5)+\text { Pemt } \\
\left(500 \mathrm{mg} / \mathrm{m}^{2}\right) \\
\text { CDBCA }(A \cup C 5)+\text { Dtx } \\
\left(75 \mathrm{mg} / \mathrm{m}^{2}\right)[\mathrm{S}]\end{array}$ & 260 & 60 \\
\hline Sandler, 2006 & USA & III & os & NSq & 2 & IIIb, IV & $\begin{array}{l}\text { ECOG } \\
0-1\end{array}$ & $\begin{array}{l}\text { CDBCA (AUC 6) + Ptx } \\
\left(200 \mathrm{mg} / \mathrm{m}^{2}\right)+\mathrm{Bev} \\
(15 \mathrm{mg} / \mathrm{kg}) \\
\text { CDBCA }(\text { AUC 6) }+\mathrm{Ptx} \\
\left(200 \mathrm{mg} / \mathrm{m}^{2}\right)[\mathrm{S}]\end{array}$ & 602 & 63 \\
\hline Scagliotti, 2002 & Italy & III & $\begin{array}{l}\text { Not } \\
\text { available }\end{array}$ & NSCLC & 3 & $\begin{array}{l}\text { IIIb, IV, } \\
\text { Rec }\end{array}$ & $\begin{array}{l}\text { ECOG } \\
0-2\end{array}$ & $\begin{array}{l}\text { CDDP }\left(75 \mathrm{mg} / \mathrm{m}^{2}\right)+\text { Gem } \\
{\left[1,250 \mathrm{mg} / \mathrm{m}^{2}(\mathrm{~d} 1,8)\right]} \\
\text { CDBCA }(\text { AUC } 6)+\mathrm{Ptx} \\
\left(225 \mathrm{mg} / \mathrm{m}^{2}\right) \\
\text { CDDP }\left(100 \mathrm{mg} / \mathrm{m}^{2}\right)+\mathrm{Vnr} \\
{\left[25 \mathrm{mg} / \mathrm{m}^{2} \text { (weekly)] [S] }\right.}\end{array}$ & 612 & 63 \\
\hline Schiller, 2002 & USA & $\begin{array}{l}\text { Not } \\
\text { available }\end{array}$ & os & NSCLC & 3 & $\begin{array}{l}\text { Illb, IV, } \\
\text { Rec }\end{array}$ & $\begin{array}{l}\text { ECOG } \\
0-2\end{array}$ & $\begin{array}{l}\left(\operatorname{CDDP}\left(75 \mathrm{mg} / \mathrm{m}^{2}\right) \text { or }\right. \\
\text { CDBCA }(A \cup C 6))+ \text { Ptx } \\
\left(135 \text { or } 225 \mathrm{mg} / \mathrm{m}^{2}\right) \\
\text { CDDP }\left(75 \mathrm{mg} / \mathrm{m}^{2}\right)+\text { Gem } \\
{\left[1,000 \mathrm{mg} / \mathrm{m}^{2}(\mathrm{~d} 1,8,15)\right]} \\
\operatorname{CDDP}\left(75 \mathrm{mg} / \mathrm{m}^{2}\right)+\operatorname{Dtx} \\
\left(75 \mathrm{mg} / \mathrm{m}^{2}\right)[\mathrm{S}]\end{array}$ & 1,207 & 63 \\
\hline
\end{tabular}

Table 1 (continued) 
Table 1 (continued)

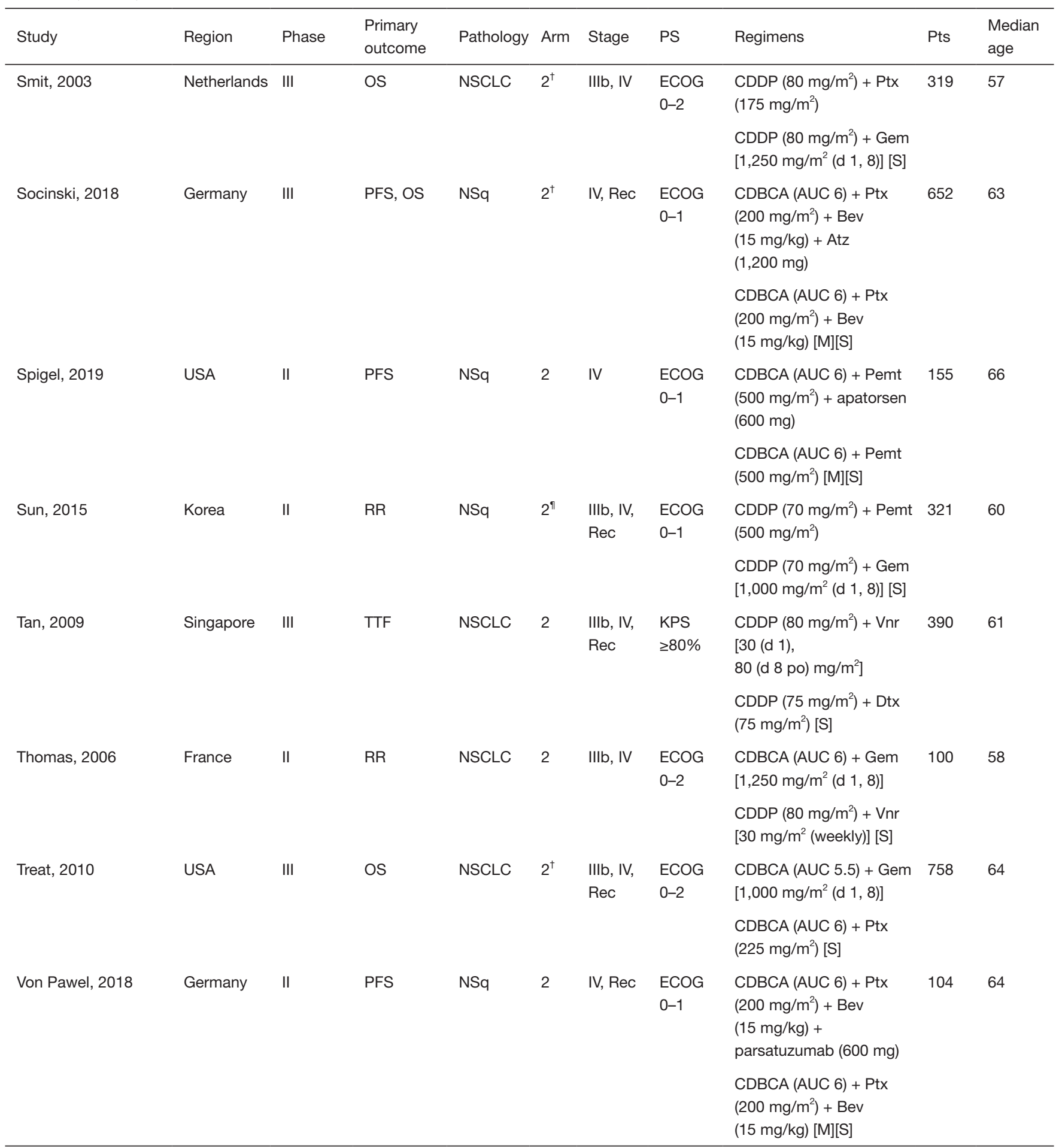

Table 1 (continued) 
Table 1 (continued)

\begin{tabular}{|c|c|c|c|c|c|c|c|c|c|c|}
\hline Study & Region & Phase & $\begin{array}{l}\text { Primary } \\
\text { outcome }\end{array}$ & Pathology & Arm & Stage & PS & Regimens & Pts & $\begin{array}{l}\text { Median } \\
\text { age }\end{array}$ \\
\hline West, 2019 & Italy & III & PFS, OS & NSq & 2 & IV & $\begin{array}{l}\text { ECOG } \\
0-1\end{array}$ & $\begin{array}{l}\text { CDBCA (AUC 6) + nabPtx } \\
{\left[100 \mathrm{mg} / \mathrm{m}^{2}(\mathrm{~d} 1,8,15)\right]+} \\
\text { Atz }(1,200 \mathrm{mg}) \\
\text { CDBCA }(\text { AUC } 6)+\text { nabPtx } \\
{\left[100 \mathrm{mg} / \mathrm{m}^{2}(\mathrm{~d} 1,8,15)\right]} \\
{[\mathrm{M}][\mathrm{S}]}\end{array}$ & 368 & 64 \\
\hline Wheatley, 2019 & USA & II & PFS & NSCLC & 2 & IIIb, IV & $\begin{array}{l}\text { ECOG } \\
0-1\end{array}$ & $\begin{array}{l}\text { CDBCA (AUC 6) + Ptx } \\
\left(200 \mathrm{mg} / \mathrm{m}^{2}\right)+\text { MEDI-575 } \\
\left(25 \mathrm{mg} / \mathrm{m}^{2}\right) \\
\text { CDBCA (AUC 6) + Ptx } \\
\left(200 \mathrm{mg} / \mathrm{m}^{2}\right)[\mathrm{M}][\mathrm{S}]\end{array}$ & 81 & $\begin{array}{l}\text { Not } \\
\text { available }\end{array}$ \\
\hline Wu, 2014 & China & III & OS & NSq & 2 & IIIb, IV & $\begin{array}{l}\text { ECOG } \\
0-1\end{array}$ & $\begin{array}{l}\operatorname{CDDP}\left(75 \mathrm{mg} / \mathrm{m}^{2}\right)+\text { Pemt } \\
\left(500 \mathrm{mg} / \mathrm{m}^{2}\right) \\
\operatorname{CDDP}\left(75 \mathrm{mg} / \mathrm{m}^{2}\right)+\mathrm{Gem} \\
{\left[1,250 \mathrm{mg} / \mathrm{m}^{2}(\mathrm{~d} 1,8)\right][\mathrm{S}]}\end{array}$ & 256 & 57 \\
\hline Yang, 2012 & China & $\begin{array}{l}\text { Not } \\
\text { available }\end{array}$ & $\mathrm{RR}$ & NSCLC & 2 & IIIb, IV & $\begin{array}{l}\text { ECOG } \\
0-2\end{array}$ & $\begin{array}{l}\text { Cdgp }\left(80 \mathrm{mg} / \mathrm{m}^{2}\right)+\text { Gem } \\
{\left[1,250 \mathrm{mg} / \mathrm{m}^{2}(\mathrm{~d} 1,8)\right]} \\
\text { CDBCA (AUC 5) + Gem } \\
{\left[1,250 \mathrm{mg} / \mathrm{m}^{2}(\mathrm{~d} 1,8)\right][\mathrm{S}]}\end{array}$ & 62 & 57 \\
\hline Zhang, 2013 & China & II & PFS & NSCLC & 2 & $\begin{array}{l}\text { Illb, IV, } \\
\text { Rec }\end{array}$ & $\begin{array}{l}\text { ECOG } \\
0-1\end{array}$ & $\begin{array}{l}\operatorname{CDDP}\left(75 \mathrm{mg} / \mathrm{m}^{2}\right)+\text { Pemt } \\
\left(500 \mathrm{mg} / \mathrm{m}^{2}\right) \\
\operatorname{CDDP}\left(75 \mathrm{mg} / \mathrm{m}^{2}\right)+\text { Gem } \\
{\left[1,000 \mathrm{mg} / \mathrm{m}^{2}(\mathrm{~d} 1,8)\right][\mathrm{S}]}\end{array}$ & 205 & 54 \\
\hline Zhou, 2015 & China & III & PFS & NSq & 2 & IV, Rec & $\begin{array}{l}\text { ECOG } \\
0-1\end{array}$ & 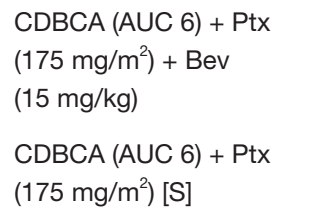 & 276 & 57 \\
\hline
\end{tabular}

Table 1 (continued) 
Table 1 (continued)

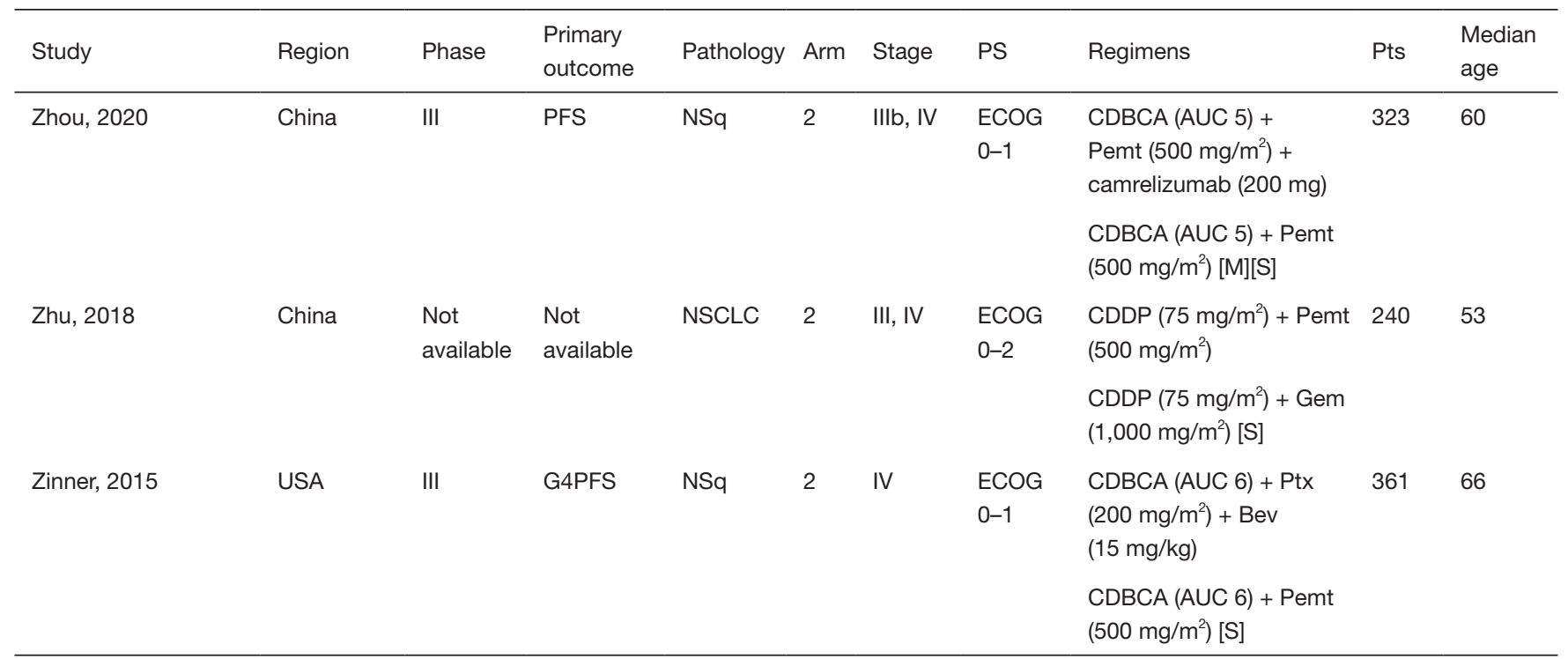

Study: first author, publication year, specific study name if available are presented; updated: updated data that were published later were available; patients: numbers of patients randomized for evaluated arms; median age: when median age (years) is not available, average age (years) is presented instead. ${ }^{\dagger}, 3>2$ (excluded); ${ }^{\ddagger}, 4>2$ (excluded); ${ }^{\S}$, two arms were regarded as one arm; ${ }^{\natural}$, stratified by TS, then randomized: the original study evaluated regimen(s) out of our concern. [M]: study incorporated in main model; [S]: study incorporated in separate model. NS, not specified; OS, overall survival; PFS, progression-free survival; QOL, quality of life; RR, response rate; DCR, disease control rate; TTP, time to progression; AE, adverse event; G3/4PFS, PFS without grade 3/4 AE; G4PFS, PFS without grade 4 AE; non-inf, primary outcome was evaluated by non-inferiority analysis; NS, not specified; NSCLC, non-small cell lung cancer; NSq, non-squamous carcinoma; Rec, recurrent; ECOG, Eastern Cooperative Oncology Group performance status; KPS, Karnofsky performance status; CDDP, cisplatin; CBDCA, carboplatin; Cdgp, nedaplatin; Dtx, docetaxel; Ptx, paclitaxel; Vnr, vinorelbine; Gem, gemcitabine; Cpt11, irinotecan; Pemt, pemetrexed; S1, Tegafur gimeracil oteracil; d, day; po, oral administration; bid, twice daily.

\section{Efficacy analysis}

Data for HRos were obtained in 26 studies with 7,142 patients (Table 1). In the main model, the HRos of 23 pairwise comparisons ranged from 0.55 to 1.64 , with a median of 0.94 . Q statistics and a test for heterogeneity did not reveal inconsistency at any level (whole network level $\mathrm{I}^{2}=0 \%$, total; $\mathrm{P}=0.348$, within designs; $\mathrm{P}=0.348$ ) (Figure 2, Figure S1). Eligible treatments were clustered into the same node. The platinum regimen + Pemb (HRos $=0.55,95 \%$ CI: $0.34-0.89, \mathrm{P}=0.015)$ showed the best OS, followed by the platinum regimen + nivolumab $(\mathrm{Niv})+$ ipilimumab (Ipi) (HR = 0.61, 95\% CI: 0.44-0.84, $\mathrm{P}=0.003$ ) (Figure $3 A$ ). The HRos of these regimens were significant against the platinum regimen. The HRos of the other regimens were not significantly different from that of the platinum regimen. The platinum regimen + atezolizumab (Atz) (HR $=0.70,95 \%$ CI: $0.45-1.08, \mathrm{P}=0.110)$ did not show superiority to the platinum regimen in terms of OS. We conducted a subgroup analysis excepting conference abstracts, which did not conflict with the main analysis with the conference abstract (Figure S2). In the separate model, the platinum regimen + Pemt + Pemb $(\mathrm{HR}=0.55,95 \%$ CI: $0.34-0.89, \mathrm{P}=0.014$ ) showed the best $\mathrm{OS}$. This regimen was significantly different in the separate model (Figure S3).

The HRpfs of the platinum regimen + Pemb were significantly decreased compared to the platinum regimen alone (HRpfs $=0.55,95 \%$ CI: $0.37-0.81, \mathrm{P}=0.003$ ) (Figure 3B). The lowest HRpfs was observed in the platinum regimen + dulanermin $(\mathrm{HRpfs}=0.40,95 \%$ CI: 0.32-0.50, $\mathrm{P}<0.001$ ), followed by the platinum regimen + sintilimab (Sint), the platinum regimen + Pemb, the platinum regimen + Niv, the platinum regimen + camrelizumab, and the platinum regimen + Atz.

\section{Safety analysis}

The lowest risk of grade III adverse events was observed in the Pemb arm $(\mathrm{OR}=0.20,95 \%$ CI: $0.11-0.37, \mathrm{P}<0.001)$ against the platinum regimen, followed by Niv, durvalumab, 


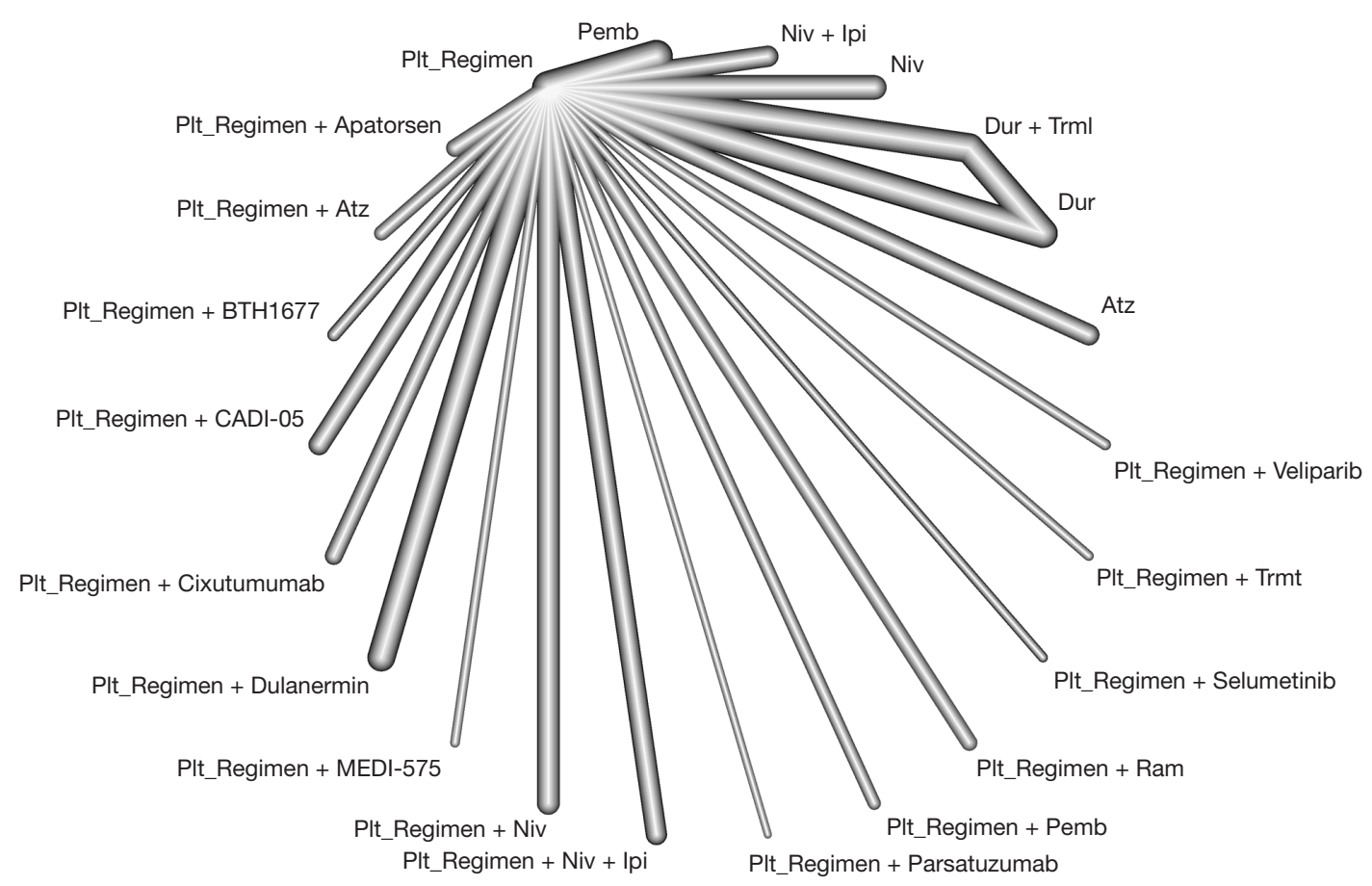

Figure 2 Network diagram for the primary endpoint, HR for OS. Addiction model, whole network level $\left(\mathrm{I}^{2}=0 \%\right.$, $\left.\mathrm{P}=0.3483\right)$. HR, hazard ratio; OS, overall survival; Plt, platinum regimen; Pemb, pembrolizumab; Niv, nivolumab; Ipi, ipilimumab; Dur, durvalumab; Trml, tremelimumab; Atz, atezolizumab; Trmt, tromethamine; Ram, ramucirumab

and Atz (Figure 3C,3D). Regarding chemotherapy-related death, there was no significant difference in regimens except the platinum regimen $+\operatorname{Sint}(\mathrm{OR}=0.31,95 \%$ CI: $0.11-0.90$, $\mathrm{P}=0.029$ ).

\section{Discussion}

We carried out the first network meta-analysis to compare regimens including cytotoxic agents, molecular targeted therapies, and ICIs for chemo-naïve incurable NSCLC with low PD-L1 expression. The network method was able to concurrently compare a variety of chemotherapy regimens. Moreover, the sufficient statistical power supported by the substantial studies ensured the validity of the results.

The immune response activated by PD-L1 inhibition is enhanced by cytotoxic chemotherapy, which reduces regulatory T-cell activity (22). Combination therapy is expected to improve the anticancer activity. Among the 22 regimens, the HRos of the platinum regimen + Pemb and the platinum regimen + Niv + Ipi were 0.55 (95\% CI: 0.34-0.89) and 0.61 (95\% CI: 0.44-0.84), respectively, with the platinum regimen alone as the reference. These regimens in this order showed the best performance in terms of OS (Figure $3 A$ ). The platinum regimen + Pemb also showed a high rank in terms of improving PFS (Figure 3B). Combination therapy is said to provide an early disease control relative to ICI monotherapy (15), preventing early disease progression. Moreover, less than half of patients with advanced NSCLC receive second-line therapy (23). Patients treated with monotherapy may miss the opportunity to receive other regimens.

The adverse events of the platinum regimen + Pemb $(\mathrm{ORae}=1.30,95 \%$ CI: $0.68-2.49)$ and the platinum regimen $+\mathrm{Niv}+\mathrm{Ipi}(\mathrm{ORae}=1.24,95 \% \mathrm{CI}: 0.52-2.95)$ were not significantly greater than those of the platinum regimen alone (Figure 3C). We recommend the platinum regimen + $\mathrm{Pemb}$ or the platinum regimen $+\mathrm{Niv}+\mathrm{Ipi}$ when the PDL1 TPS is $1-49 \%$. By contrast, there was no significant difference between the HRos of ICI monotherapy and the platinum regimen; therefore, ICI monotherapy is not recommended. However, ICI monotherapy tends to have a low risk of adverse events. The ORae with Pemb 

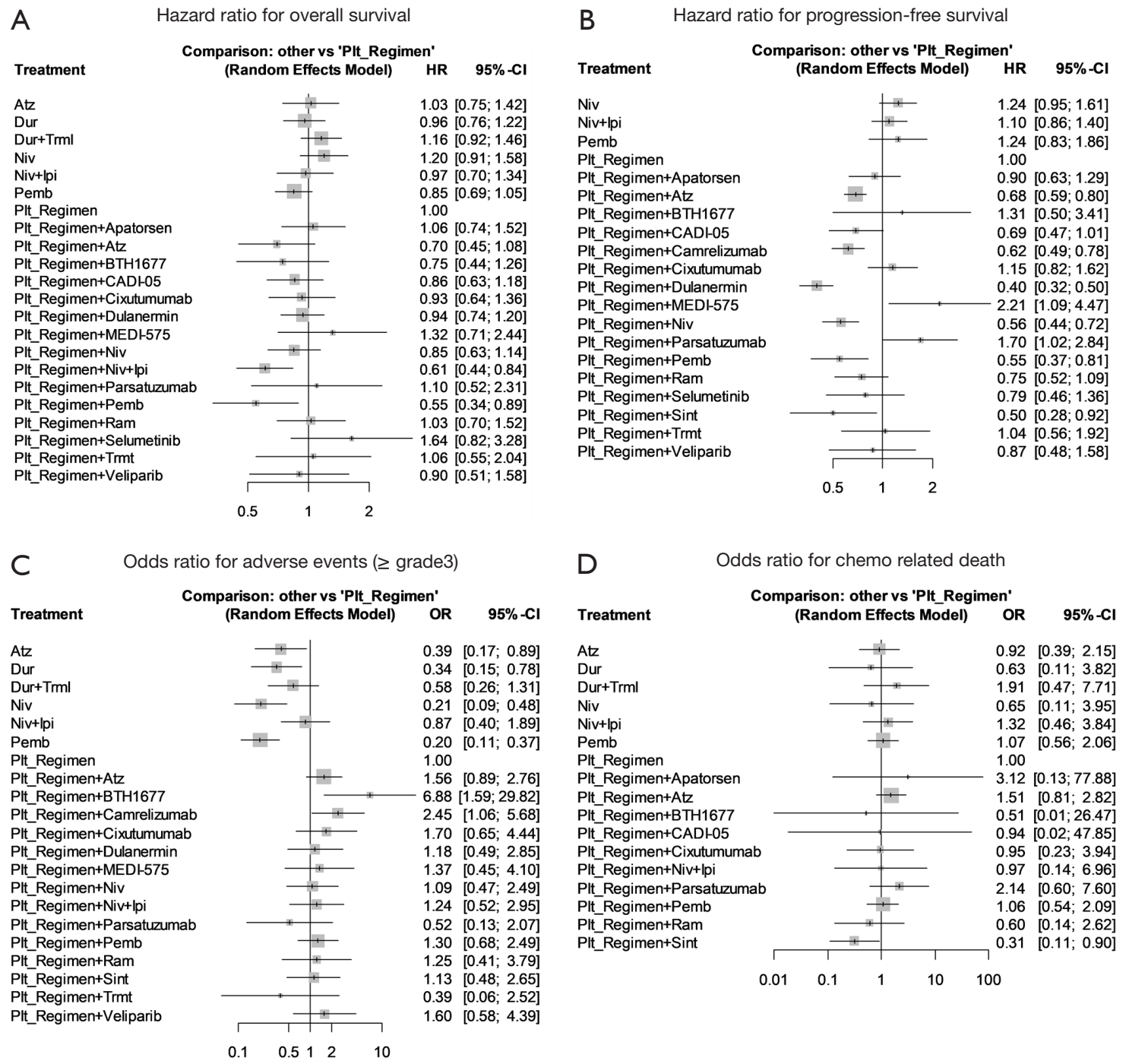

Figure 3 Forest plots for primary and secondary outcomes in main model. (A) HR for OS. (B) HR for PFS. (C) OR for adverse events ( $\geq$ grade 3). (D) OR for chemo related death. HR, hazard ratio; OS, overall survival; PFS, progression-free survival; OR, odds ratio; Plt, platinum regimen; Pemb, pembrolizumab; Niv, nivolumab; Ipi, ipilimumab; Dur, durvalumab; Trml, tremelimumab; Atz, atezolizumab; Trmt, tromethamine; Ram, ramucirumab; Sint, sintilimab; HR, hazard ratio; OR, odds ratios; CI, confidence interval.

monotherapy was the lowest in this study $($ ORae $=0.20$, 95\% CI: 0.11-0.37) (Figure 3C). These regimens can be considered if the patients are elderly and have a low PS.

The platinum regimen + dulanermin did not have a significant effect on OS compared to the platinum regimen, but this regimen had the lowest HRpfs. Dulanermin is recombinant TRAIL/Apo2L, a novel molecular target, which can induce apoptosis in tumor cells. However, dulanermin is administered intravenously on days 1 to 14 in a cycle until disease progression.
When a patient fulfills the criteria of this study, the Japanese Lung Cancer Society Guideline (4) recommends adding Atz to the platinum regimen in accordance with the guidelines of the American Society of Clinical Oncology, National Comprehensive Cancer Network, and European Society for Medical Oncology. Our study did not show a significant effect of the Atz regimen on OS $(\mathrm{HR}=0.70$, 95\% CI: 0.45-1.08) (Figure 3A). Impower 150 (24), which compared platinum + Ptx + Bev + Atz and platinum + Ptx + Bev, indicated that adding Atz was effective in terms of PFS 
(HRpfs $=0.68,95 \%$ CI: 0.56-0.82, $\mathrm{P}<0.001)$, but the HRos in patients with low PD-L1 expression (1-49\%) was not shown.

There were some limitations to our study. First, most of the evaluated original trials had a high risk of bias, as judged by the Cochrane tool. Unfortunately, in practical terms, it is difficult to conduct a double-blinded trial without sponsorship, and we believe that these factors do not largely reduce the credibility. Second, because the main model regarded each platinum regimen as identical, the results may not be accurate. However, we believe that the two models have similar consequences. Thirdly, different PD-L1 assays were usually selected for pathological specimen based on the ICI drug selection. Furthermore, there is no universally accepted judgement for PD-L1 positivity. As a result, even though we tried to find out original studies with cutoff value of PD-L1 $1 \%$ and 50\%, such cutoff values might be slightly different among studies (25). In conclusion, we conducted a systematic review and network meta-analysis examining ICIs in patients with non-squamous NSCLC with low PD-L1 expression. Based on 20,257 NSCLC patients constituting 59 RCTs, the platinum regimen + Pemb and the platinum regimen + Niv + Ipi seem to be reasonable first-line regimens for non-squamous NSCLC with a PDL1 TPS $1-49 \%$.

\section{Acknowledgments}

We would like to thank Editage (www.editage.com) for English language editing.

Funding: None.

\section{Footnote}

Reporting Checklist: The authors have completed the PRISMA NMA reporting checklist. Available at https:// dx.doi.org/10.21037/tlcr-21-419

Peer Review File: Available at https://dx.doi.org/10.21037/ tlcr-21-419

Conflicts of Interest: All authors have completed the ICMJE uniform disclosure form (available at https:// dx.doi.org/10.21037/tlcr-21-419). Dr. NH has received personal fee from Taiho Pharmaceutical and research grant from Taiho Pharmaceutical outside of the work. Dr. KW has received personal fee from AstraZeneca,
Ono Pharmaceutical and Boehringer Ingelheim outside of the work. Dr. YH has received personal fee from AstraZeneca and Boehringer Ingelheim outside of the work. Dr. NK has received personal fee from Chugai Pharmaceutical, AstraZeneca, Boehringer Ingelheim, Sanofi, Ono Pharmaceutical, MSD, Bristol Myers Squibb, Eli Lilly, Kyowa Kirin and research grant from Chugai Pharmaceutical, Boehringer Ingelheim, MSD, Eli Lilly, Kyowa Kirin, Daiichi Sankyo, Pfizer outside of the work. Dr. TK has received personal fee from Chugai Pharmaceutical, AstraZeneca, Bristol Myers Squibb, Eli Lilly, Taiho Pharmaceutical, Chugai Pharmaceutical, Daiichi Sankyo, Sanofi, Pfizer and research grant from MSD, Chugai Pharmaceutical, Eli Lilly, Taiho Pharmaceutical, Chugai Pharmaceutical, Daiichi Sankyo, Pfizer, Shionogi outside of the work. The other authors have no conflicts of interest to declare.

Ethical Statement: The authors are accountable for all aspects of the work in ensuring that questions related to the accuracy or integrity of any part of the work are appropriately investigated and resolved.

Open Access Statement: This is an Open Access article distributed in accordance with the Creative Commons Attribution-NonCommercial-NoDerivs 4.0 International License (CC BY-NC-ND 4.0), which permits the noncommercial replication and distribution of the article with the strict proviso that no changes or edits are made and the original work is properly cited (including links to both the formal publication through the relevant DOI and the license). See: https://creativecommons.org/licenses/by-nc-nd/4.0/.

\section{References}

1. Jemal A, Bray F, Center MM, et al. Global cancer statistics. CA Cancer J Clin 2011;61:69-90.

2. Mahoney KM, Rennert PD, Freeman GJ. Combination cancer immunotherapy and new immunomodulatory targets. Nat Rev Drug Discov 2015;14:561-84.

3. Buchbinder EI, Desai A. CTLA-4 and PD-1 pathways: similarities, differences, and implications of their inhibition. Am J Clin Oncol 2016;39:98-106.

4. Akamatsu H, Ninomiya K, Kenmotsu H, et al. The Japanese Lung Cancer Society Guideline for nonsmall cell lung cancer, stage IV. Int J Clin Oncol 2019;24:731-70.

5. Hanna NH, Schneider BJ, Temin S, et al. Therapy for 
stage IV Non-Small-Cell lung cancer without driver alterations: $\mathrm{ASCO}$ and $\mathrm{OH}(\mathrm{CCO})$ joint guideline update. J Clin Oncol 2020;38:1608-32.

6. National Comprehensive Cancer Network. NCCN Clinical Practice Guidelines in Oncology: non-small cell lung cancer. 2020. Available online: https://www.nccn.org (accessed 14 April 2021).

7. European Society for Medical Oncology. Metastatic nonsmall cell lung cancer: ESMO Clinical Practice Guidelines for diagnosis, treatment and follow-up: updated version published 15. 2020. Available online: https://www.esmo. org (accessed 13 April 2021).

8. Rotow J, Bivona TG. Understanding and targeting resistance mechanisms in NSCLC. Nat Rev Cancer 2017;17:637-58.

9. Horita N, Nagashima A, Nakashima K, et al. The best platinum regimens for chemo-naive incurable nonsmall cell lung cancer: network meta-analysis. Sci Rep 2017;7:13185.

10. Hutton B, Salanti G, Caldwell DM, et al. The PRISMA extension statement for reporting of systematic reviews incorporating network meta-analyses of health care interventions: checklist and explanations. Ann Intern Med 2015;162:777-84.

11. University Hospital Medical Information Network (UMIN) Center. Available online: https://www.umin.ac.jp/ ctr/ctr_regist.htm (accessed 15 October 2020).

12. Hotta K, Matsuo K, Ueoka H, et al. Meta-analysis of randomized clinical trials comparing Cisplatin to Carboplatin in patients with advanced non-small-cell lung cancer. J Clin Oncol 2004;22:3852-9.

13. Griesinger F, Korol EE, Kayaniyil S, et al. Efficacy and safety of first-line carboplatin-versus cisplatin-based chemotherapy for non-small cell lung cancer: a metaanalysis. Lung Cancer 2019;135:196-204.

14. Gandhi L, Rodríguez-Abreu D, Gadgeel S, et al. Pembrolizumab plus chemotherapy in metastatic nonsmall-cell lung cancer. N Engl J Med 2018;378:2078-92.

15. Hellmann MD, Paz-Ares L, Bernabe Caro R, et al. Nivolumab plus ipilimumab in advanced non-small-cell lung cancer. N Engl J Med 2019;381:2020-31.

16. Higgins JP, Altman DG, Gøtzsche PC, et al. The Cochrane Collaboration's tool for assessing risk of bias in randomised trials. BMJ 2011;343:d5928.

17. Atkinson TM, Ryan SJ, Bennett AV, et al. The association between clinician-based common terminology criteria for adverse events (CTCAE) and patient-reported outcomes (PRO): a systematic review. Support Care Cancer 2016;24:3669-76.

18. Eisenhauer EA, Therasse P, Bogaerts J, et al. New response evaluation criteria in solid tumours: revised RECIST guideline (version 1.1). Eur J Cancer 2009;45:228-47.

19. Parmar MK, Torri V, Stewart L. Extracting summary statistics to perform meta-analyses of the published literature for survival endpoints. Stat Med 1998;17:2815-34.

20. Salanti G. Indirect and mixed-treatment comparison, network, or multiple-treatments meta-analysis: many names, many benefits, many concerns for the next generation evidence synthesis tool. Res Synth Methods 2012;3:80-97.

21. Rucker G. Package "netmeta": network meta-analysis using frequentist methods. 2016. Available online: https:// cran.r-project.org/web/packages/netmeta/netmeta. pdf\#search $=($ accessed 14 April 2021).

22. Zitvogel L, Galluzzi L, Smyth MJ, et al. Mechanism of action of conventional and targeted anticancer therapies: reinstating immunosurveillance. Immunity 2013;39:74-88.

23. Davies J, Patel M, Gridelli C, et al. Real-world treatment patterns for patients receiving second-line and thirdline treatment for advanced non-small cell lung cancer: a systematic review of recently published studies. PLoS One 2017;12:e0175679.

24. Socinski MA, Jotte RM, Cappuzzo F, et al. Atezolizumab for first-line treatment of metastatic nonsquamous NSCLC. N Engl J Med 2018;378:2288-301.

25. Paver EC, Cooper WA, Colebatch AJ, et al. Programmed death ligand-1 (PD-L1) as a predictive marker for immunotherapy in solid tumours: a guide to immunohistochemistry implementation and interpretation. Pathology 2021;53:141-56.

Cite this article as: Fukuda N, Horita N, Katakura S, Namkoong H, Kaneko A, Somekawa K, Tagami Y, Watanabe K, Hara Y, Kobayashi N, Kaneko T. The best regimens for chemo-naïve incurable non-squamous non-small cell lung cancer with a programmed death-ligand 1 , tumor proportion score 1-49\%: a network meta-analysis. Transl Lung Cancer Res 2021;10(8):3550-3566. doi: 10.21037/tlcr-21-419 


\section{Appendix 1}

\section{The amendments to information in the protocol}

* OR of objective response rate was not evaluated;

* The populations with TPS 0\% were excluded.

\section{Appendix 2}

\section{Search formulas}

\section{MEDLINE}

(non-small OR squamous OR adenocarcinoma OR non-squamous OR NSCLC) AND (lung cancer OR lung carcinoma OR lung malignancy OR lung tumor OR NSCLC) AND (advanced OR metastasis OR recurrent OR recurrence OR inoperable OR relapsed OR incurable OR stage 3 OR stage 3a OR stage 3b OR stage III OR stage IIIa OR stage IIIb OR stage 4 OR stage 4a OR stage 4b OR stage IV OR stage IVa OR stage IVb) AND (naïve OR untreated OR chemo naïve OR chemonaïve OR non-treated OR nontreated OR first-line OR front-line OR initial treatment OR "previously not treated") AND (randomised[title] OR randomized[title] OR randomly OR phase 3[title] OR phase III[title] OR RCT[title] OR (nejm AND (randomized OR randomly OR phase 3 OR phase III OR RCT))).

\section{Appendix 3}

\section{References}

1. Belani CP, Chakraborty BC, Modi RI, et al. A randomized trial of TLR-2 agonist CADI-05 targeting desmocollin-3 for advanced non-small-cell lung cancer. Ann Oncol 2017;28:298-304.

2. Bennouna J, Havel L, Krzakowski M, et al. Oral vinorelbine plus cisplatin as first-line chemotherapy in nonsquamous non-small-cell lung cancer: final results of an International randomized phase II study (NAVotrial 01). Clin Lung Cancer 2014;15:258-65.

3. Carbone DP, Reck M, Paz-Ares L, et al. First-Line Nivolumab in Stage IV or Recurrent Non-Small-Cell Lung Cancer. N Engl J Med 2017;376:2415-26.

4. Chang JW, Tsao TC, Yang CT, et al. A randomized study of gemcitabine plus cisplatin and vinorelbine plus cisplatin in patients with advanced non-small-cell lung cancer. Chang Gung Med J 2008;31:559-66.

5. Chen YM, Perng RP, Shih JF, et al. A randomised phase II study of weekly paclitaxel or vinorelbine in combination with cisplatin against inoperable non-small-cell lung cancer previously untreated. Br J Cancer 2004;90:359-65.

6. Chen YM, Perng RP, Shih JF, et al. A randomized phase II study of docetaxel or vinorelbine in combination with cisplatin against inoperable, chemo-naïve non-small-cell lung cancer in Taiwan. Lung Cancer 2007;56:363-9.

7. Comella P, Frasci G, Panza N, et al. Randomized trial comparing cisplatin, gemcitabine, and vinorelbine with either cisplatin and gemcitabine or cisplatin and vinorelbine in advanced non-small-cell lung cancer: interim analysis of a phase III trial of the Southern Italy Cooperative Oncology Group. J Clin Oncol 2000;18:1451-7.

8. Doebele RC, Spigel D, Tehfe M, et al. Phase 2, randomized, open-label study of ramucirumab in combination with first-line pemetrexed and platinum chemotherapy in patients with nonsquamous, advanced/metastatic non-small cell lung cancer. Cancer 2015;121:883-92.

9. Douillard JY, Gervais R, Dabouis G, et al. Sequential two-line strategy for stage IV non-small-cell lung cancer: docetaxelcisplatin versus vinorelbine-cisplatin followed by cross-over to single-agent docetaxel or vinorelbine at progression: final results of a randomised phase II study. Ann Oncol 2005;16:81-9. 
10. Edelman MJ, Clark JI, Chansky K, et al. Randomized phase II trial of sequential chemotherapy in advanced non-small cell lung cancer (SWOG 9806): carboplatin/gemcitabine followed by paclitaxel or cisplatin/vinorelbine followed by docetaxel. Clin Cancer Res 2004;10:5022-6.

11. Engel-Riedel W, Lowe J, Mattson P, et al. A randomized, controlled trial evaluating the efficacy and safety of BTH1677 in combination with bevacizumab, carboplatin, and paclitaxel in first-line treatment of advanced non-small cell lung cancer. J Immunother Cancer 2018;6:16.

12. Fossella F, Pereira JR, von Pawel J, et al. Randomized, multinational, phase III study of docetaxel plus platinum combinations versus vinorelbine plus cisplatin for advanced non-small-cell lung cancer: the TAX 326 study group. J Clin Oncol 2003;21:3016-24.

13. Galetta D, Cinieri S, Pisconti S, et al. Cisplatin/Pemetrexed Followed by Maintenance Pemetrexed Versus Carboplatin/ Paclitaxel/Bevacizumab Followed by Maintenance Bevacizumab in Advanced Nonsquamous Lung Cancer: The GOIM (Gruppo Oncologico Italia Meridionale) ERACLE Phase III Randomized Trial. Clin Lung Cancer 2015;16:262-73.

14. Gandhi L, Rodríguez-Abreu D, Gadgeel S, et al. Pembrolizumab plus Chemotherapy in Metastatic Non-Small-Cell Lung Cancer. N Engl J Med 2018;378:2078-92.

15. Garon EB, Neidhart JD, Gabrail NY, et al. A randomized Phase II trial of the tumor vascular disrupting agent CA4P (fosbretabulin tromethamine) with carboplatin, paclitaxel, and bevacizumab in advanced nonsquamous non-small-cell lung cancer. Onco Targets Ther 2016;9:7275-83.

16. Gebbia V, Lorusso V, Galetta D, et al. First-line cisplatin with docetaxel or vinorelbine in patients with advanced non-smallcell lung cancer: a quality of life directed phase II randomized trial of Gruppo Oncologico Italia Meridionale. Lung Cancer 2010;69:218-24.

17. Gebbia V, Galetta D, Caruso M, et al. Gemcitabine and cisplatin versus vinorelbine and cisplatin versus ifosfamide+gemcitabine followed by vinorelbine and cisplatin versus vinorelbine and cisplatin followed by ifosfamide and gemcitabine in stage IIIB-IV non small cell lung carcinoma: a prospective randomized phase III trial of the Gruppo Oncologico Italia Meridionale. Lung Cancer 2003;39:179-89.

18. Grønberg BH, Bremnes RM, Fløtten O, et al. Phase III study by the Norwegian lung cancer study group: pemetrexed plus carboplatin compared with gemcitabine plus carboplatin as first-line chemotherapy in advanced non-small-cell lung cancer. J Clin Oncol 2009;27:3217-24.

19. Harada T, Udagawa H, Sugiyama E, et al. P1.01-33 Randomized Phase 2 Study Comparing CBDCA+PTX+BEV and CDDP+PEM+BEV in Treatment-Naïve Advanced Non-Sq NSCLC (CLEAR study). J Thorac Oncol 2018;13:S472-3.

20. Helbekkmo N, Sundstrøm SH, Aasebø U, et al. Vinorelbine/carboplatin vs gemcitabine/carboplatin in advanced NSCLC shows similar efficacy, but different impact of toxicity. Br J Cancer 2007;97:283-9.

21. Hellmann MD, Paz-Ares L, Bernabe Caro R, et al. Nivolumab plus Ipilimumab in Advanced Non-Small-Cell Lung Cancer. N Engl J Med 2019;381:2020-31.

22. Herbst RS, Giaccone G, de Marinis F, et al. Atezolizumab for First-Line Treatment of PD-L1-Selected Patients with NSCLC. N Engl J Med 2020;383:1328-39.

23. Kader YA, Le Chevalier T, El-Nahas T, et al. Comparative study analyzing survival and safety of bevacizumab/carboplatin/ paclitaxel and cisplatin/pemetrexed in chemotherapy-naïve patients with advanced non-squamous bronchogenic carcinoma not harboring EGFR mutation. Onco Targets Ther 2013;6:803-9.

24. Kaira K, Imai H, Souma R, et al. An Exploratory Randomized Phase II Trial Comparing CDDP Plus S-1 With Bevacizumab and CDDP Plus Pemetrexed With Bevacizumab Against Patients With Advanced Non-squamous Non-small Cell Lung Cancer. Anticancer Res 2019;39:2483-91.

25. Kawahara M, Atagi S, Komuta K, et al. Carboplatin plus either docetaxel or paclitaxel for Japanese patients with advanced nonsmall cell lung cancer. Anticancer Res 2013;33:4631-7.

26. Khodadad K, Khosravi A, Esfahani-Monfared Z, et al. Comparing docetaxel plus Cisplatin with Paclitaxel plus Carboplatin in chemotherapy-naïve patients with advanced non-small-cell lung cancer: a single institute study. Iran J Pharm Res 2014;13:575-81.

27. Kubota K, Sakai H, Katakami N, et al. A randomized phase III trial of oral S-1 plus cisplatin versus docetaxel plus cisplatin in Japanese patients with advanced non-small-cell lung cancer: TCOG0701 CATS trial. Ann Oncol 2015;26:1401-8.

28. Langer CJ, Gadgeel SM, Borghaei H, et al. Carboplatin and pemetrexed with or without pembrolizumab for advanced, non- 
squamous non-small-cell lung cancer: a randomised, phase 2 cohort of the open-label KEYNOTE-021 study. Lancet Oncol 2016;17:1497-508.

29. Lee JS, Sugawara S, Kang JH, et al. LBA54 Randomized phase III trial of nivolumab in combination with carboplatin, paclitaxel, and bevacizumab as first-line treatment for patients with advanced or recurrent non-squamous NSCLC. Ann Oncol 2020;31:S1184-5.

30. Martoni A, Marino A, Sperandi F, et al. Multicentre randomised phase III study comparing the same dose and schedule of cisplatin plus the same schedule of vinorelbine or gemcitabine in advanced non-small cell lung cancer. Eur J Cancer 2005;41:81-92.

31. Melosky B, Bradbury P, Tu D, et al. Selumetinib in patients receiving standard pemetrexed and platinum-based chemotherapy for advanced or metastatic KRAS wildtype or unknown non-squamous non-small cell lung cancer: A randomized, multicenter, phase II study. Canadian Cancer Trials Group (CCTG) IND.219. Lung Cancer 2019;133:48-55.

32. Minami S, Kijima T, Shiroyama T, et al. Randomized Phase II trial of paclitaxel and carboplatin followed by gemcitabine switch-maintenance therapy versus gemcitabine and carboplatin followed by gemcitabine continuation-maintenance therapy in previously untreated advanced non-small cell lung cancer. BMC Res Notes 2013;6:3.

33. Mok TSK, Wu YL, Kudaba I, et al. Pembrolizumab versus chemotherapy for previously untreated, PD-L1-expressing, locally advanced or metastatic non-small-cell lung cancer (KEYNOTE-042): a randomised, open-label, controlled, phase 3 trial. Lancet 2019;393:1819-30.

34. Niho S, Kunitoh H, Nokihara H, et al. Randomized phase II study of first-line carboplatin-paclitaxel with or without bevacizumab in Japanese patients with advanced non-squamous non-small-cell lung cancer. Lung Cancer 2012;76:362-7.

35. Novello S, Scagliotti G, de Castro G Jr, et al. An Open-Label, Multicenter, Randomized, Phase II Study of Cisplatin and Pemetrexed With or Without Cixutumumab (IMC-A12) as a First-Line Therapy in Patients With Advanced Nonsquamous Non-Small Cell Lung Cancer. J Thorac Oncol 2017;12:383-9.

36. Ohe Y, Ohashi Y, Kubota K, et al. Randomized phase III study of cisplatin plus irinotecan versus carboplatin plus paclitaxel, cisplatin plus gemcitabine, and cisplatin plus vinorelbine for advanced non-small-cell lung cancer: Four-Arm Cooperative Study in Japan. Ann Oncol 2007;18:317-23.

37. Okamoto I, Yoshioka H, Morita S, et al. Phase III trial comparing oral S-1 plus carboplatin with paclitaxel plus carboplatin in chemotherapy-naïve patients with advanced non-small-cell lung cancer: results of a west Japan oncology group study. J Clin Oncol 2010;28:5240-6.

38. Ouyang X, Shi M, Jie F, et al. Phase III study of dulanermin (recombinant human tumor necrosis factor-related apoptosisinducing ligand/Apo2 ligand) combined with vinorelbine and cisplatin in patients with advanced non-small-cell lung cancer. Invest New Drugs 2018;36:315-22.

39. Papadimitrakopoulou V, Cobo M, Bordoni R, et al. IMpower132: PFS and safety results with 1L atezolizumab+ carboplatin/ cisplatin+ pemetrexed in stage IV non-squamous NSCLC. J Thorac Oncol 2018;13:S332-3.

40. Patel JD, Socinski MA, Garon EB, et al. PointBreak: a randomized phase III study of pemetrexed plus carboplatin and bevacizumab followed by maintenance pemetrexed and bevacizumab versus paclitaxel plus carboplatin and bevacizumab followed by maintenance bevacizumab in patients with stage IIIB or IV nonsquamous non-small-cell lung cancer. J Clin Oncol 2013;31:4349-57.

41. Paz-Ares L, Ciuleanu TE, Cobo M, et al. First-line nivolumab plus ipilimumab combined with two cycles of chemotherapy in patients with non-small-cell lung cancer (CheckMate 9LA): an international, randomised, open-label, phase 3 trial. Lancet Oncol 2021;22:198-211.

42. Ramalingam SS, Blais N, Mazieres J, et al. Randomized, Placebo-Controlled, Phase II Study of Veliparib in Combination with Carboplatin and Paclitaxel for Advanced/Metastatic Non-Small Cell Lung Cancer. Clin Cancer Res 2017;23:1937-44.

43. Reck M, von Pawel J, Zatloukal P, et al. Phase III trial of cisplatin plus gemcitabine with either placebo or bevacizumab as firstline therapy for nonsquamous non-small-cell lung cancer: AVAil. J Clin Oncol 2009;27:1227-34.

44. Rizvi NA, Cho BC, Reinmuth N, et al. Durvalumab With or Without Tremelimumab vs Standard Chemotherapy in Firstline Treatment of Metastatic Non-Small Cell Lung Cancer: The MYSTIC Phase 3 Randomized Clinical Trial. JAMA Oncol 2020;6:661-74.

45. Rodrigues-Pereira J, Kim JH, Magallanes M, et al. A randomized phase 3 trial comparing pemetrexed/carboplatin and docetaxel/ carboplatin as first-line treatment for advanced, nonsquamous non-small cell lung cancer. J Thorac Oncol 2011;6:1907-14. 
46. Sandler A, Gray R, Perry MC, et al. Paclitaxel-carboplatin alone or with bevacizumab for non-small-cell lung cancer. N Engl J Med 2006;355:2542-50.

47. Scagliotti GV, Parikh P, von Pawel J, et al. Phase III study comparing cisplatin plus gemcitabine with cisplatin plus pemetrexed in chemotherapy-naive patients with advanced-stage non-small-cell lung cancer. J Clin Oncol 2008;26:3543-51.

48. Scagliotti GV, De Marinis F, Rinaldi M, et al. Phase III randomized trial comparing three platinum-based doublets in advanced non-small-cell lung cancer. J Clin Oncol 2002;20:4285-91.

49. Schiller JH, Harrington D, Belani CP, et al. Comparison of four chemotherapy regimens for advanced non-small-cell lung cancer. N Engl J Med 2002;346:92-8.

50. Smit EF, van Meerbeeck JP, Lianes $P$, et al. Three-arm randomized study of two cisplatin-based regimens and paclitaxel plus gemcitabine in advanced non-small-cell lung cancer: a phase III trial of the European Organization for Research and Treatment of Cancer Lung Cancer Group--EORTC 08975. J Clin Oncol 2003;21:3909-17.

51. Socinski MA, Jotte RM, Cappuzzo F, et al. Atezolizumab for First-Line Treatment of Metastatic Nonsquamous NSCLC. N Engl J Med 2018;378:2288-301.

52. Spigel DR, Shipley DL, Waterhouse DM, et al. A Randomized, Double-Blinded, Phase II Trial of Carboplatin and Pemetrexed with or without Apatorsen (OGX-427) in Patients with Previously Untreated Stage IV Non-Squamous-Non-Small-Cell Lung Cancer: The SPRUCE Trial. Oncologist 2019;24:e1409-16.

53. Sun JM, Ahn JS, Jung SH, et al. Pemetrexed Plus Cisplatin Versus Gemcitabine Plus Cisplatin According to Thymidylate Synthase Expression in Nonsquamous Non-Small-Cell Lung Cancer: A Biomarker-Stratified Randomized Phase II Trial. J Clin Oncol 2015;33:2450-6.

54. Tan EH, Rolski J, Grodzki T, et al. Global Lung Oncology Branch trial 3 (GLOB3): final results of a randomised multinational phase III study alternating oral and i.v. vinorelbine plus cisplatin versus docetaxel plus cisplatin as first-line treatment of advanced non-small-cell lung cancer. Ann Oncol 2009;20:1249-56.

55. Thomas P, Robinet G, Gouva S, et al. Randomized multicentric phase II study of carboplatin/gemcitabine and cisplatin/ vinorelbine in advanced non-small cell lung cancer GFPC 99-01 study (Groupe français de pneumo-cancérologie). Lung Cancer 2006;51:105-14.

56. Treat JA, Gonin R, Socinski MA, et al. A randomized, phase III multicenter trial of gemcitabine in combination with carboplatin or paclitaxel versus paclitaxel plus carboplatin in patients with advanced or metastatic non-small-cell lung cancer. Ann Oncol 2010;21:540-7.

57. von Pawel J, Spigel DR, Ervin T, et al. Randomized Phase II Trial of Parsatuzumab (Anti-EGFL7) or Placebo in Combination with Carboplatin, Paclitaxel, and Bevacizumab for First-Line Nonsquamous Non-Small Cell Lung Cancer. Oncologist 2018;23:654-e58.

58. West H, McCleod M, Hussein M, et al. Atezolizumab in combination with carboplatin plus nab-paclitaxel chemotherapy compared with chemotherapy alone as first-line treatment for metastatic non-squamous non-small-cell lung cancer (IMpower130): a multicentre, randomised, open-label, phase 3 trial. Lancet Oncol 2019;20:924-37.

59. Wheatley-Price P, Gadgeel S, Takahashi T, et al. Phase 1b/2 Randomized Study of MEDI-575 in Combination With Carboplatin Plus Paclitaxel Versus Carboplatin Plus Paclitaxel Alone in Adult Patients With Previously Untreated Advanced Non-Small-Cell Lung Cancer. Clin Lung Cancer 2019;20:e362-8.

60. Wu YL, Zhang L, Fan Y, et al. Randomized clinical trial of pembrolizumab vs chemotherapy for previously untreated Chinese patients with PD-L1-positive locally advanced or metastatic non-small-cell lung cancer: KEYNOTE-042 China Study. Int J Cancer 2021;148:2313-20.

61. Wu YL, Lu S, Cheng Y, et al. Efficacy and safety of pemetrexed/cisplatin versus gemcitabine/cisplatin as first-line treatment in Chinese patients with advanced nonsquamous non-small cell lung cancer. Lung Cancer 2014;85:401-7.

62. Yang Y, Wang Z, Fang J, et al. Efficacy and Safety of Sintilimab Plus Pemetrexed and Platinum as First-Line Treatment for Locally Advanced or Metastatic Nonsquamous NSCLC: a Randomized, Double-Blind, Phase 3 Study (Oncology pRogram by InnovENT anti-PD-1-11). J Thorac Oncol 2020;15:1636-46.

63. Yang JJ, Zhou Q, Liao RQ, et al. Nedaplatin/Gemcitabine Versus Carboplatin/Gemcitabine in Treatment of Advanced Nonsmall Cell Lung Cancer: A Randomized Clinical Trial. Chin J Cancer Res 2012;24:97-102.

64. Zhang X, Lu J, Xu J, et al. Pemetrexed plus platinum or gemcitabine plus platinum for advanced non-small cell lung cancer: final survival analysis from a multicentre randomized phase II trial in the East Asia region and a meta-analysis. Respirology 
2013;18:131-9.

65. Zhou C, Wu YL, Chen G, et al. BEYOND: A Randomized, Double-Blind, Placebo-Controlled, Multicenter, Phase III Study of First-Line Carboplatin/Paclitaxel Plus Bevacizumab or Placebo in Chinese Patients With Advanced or Recurrent Nonsquamous Non-Small-Cell Lung Cancer. J Clin Oncol 2015;33:2197-204.

66. Zhou C, Chen G, Huang Y, et al. Camrelizumab plus carboplatin and pemetrexed versus chemotherapy alone in chemotherapynaive patients with advanced non-squamous non-small-cell lung cancer (CameL): a randomised, open-label, multicentre, phase 3 trial. Lancet Respir Med 2021;9:305-14.

67. Zhu YM, Gan YL, Xu HY, et al. Clinical effectiveness of pemetrexed combined with cisplatin chemotherapy for advanced and maintenance treatment for patients with non-small-cell lung cancer. Eur Rev Med Pharmacol Sci 2018;22:1943-7.

68. Zinner RG, Obasaju CK, Spigel DR, et al. PRONOUNCE: randomized, open-label, phase III study of first-line pemetrexed + carboplatin followed by maintenance pemetrexed versus paclitaxel + carboplatin + bevacizumab followed by maintenance bevacizumab in patients ith advanced nonsquamous non-small-cell lung cancer. J Thorac Oncol 2015;10:134-42.

\section{Appendix 4}

\section{Characteristics of the included studies}

The included studies were reported in a variety of countries worldwide. The United States of America had the most included studies (20 studies). The articles were published between 2000 and 2020. Among 68 reports, 37 were phase III studies, 26 evaluated OS as the primary endpoint, 43 included ECOG $0-1$ cases. We regarded 3 studies as three-arm studies, 1 study as a four-arm study, and the other 64 as two-arm studies. 
Table S1 The Cochrane Risk of Bias evaluation sheet

\begin{tabular}{|c|c|c|c|c|c|c|}
\hline Study & Selection bias & Performance bias & Detection bias & Attrition bias & Reporting bias & Other bias \\
\hline Belani, 2017 & Low & High & Low & Low & Low & Low \\
\hline Bennouna, 2014 & Unclear & High & Low & Low & Low & High \\
\hline Carbone, 2017 & Low & High & Low & Low & Low & High \\
\hline Chang, 2008 & Unclear & High & Low & Low & Low & Low \\
\hline Chen, 2007 & Unclear & High & Low & Low & High & Low \\
\hline Chen, 2004 & Unclear & High & Low & Low & Low & Low \\
\hline Comella, 2000 & Low & High & Low & Low & Low & Low \\
\hline Doebele, 2015 & Low & High & Low & Low & Low & High \\
\hline Douillard, 2005 & Unclear & High & Low & Low & Low & High \\
\hline Edelman, 2004 & Low & High & Low & Low & Low & Low \\
\hline Engle-Riedel, 2018 & Low & High & Low & Low & Low & Low \\
\hline Fossella, 2003 & Low & High & Low & Low & Low & High \\
\hline Galetta, 2015 & Unclear & High & Low & Low & Low & Low \\
\hline Gandhi, 2018 & Low & Low & Low & Low & Low & High \\
\hline Garon, 2016 & Low & High & Low & Low & Low & High \\
\hline Gebbia, 2010 & Low & High & Low & Low & Low & Low \\
\hline Gebbia, 2003 & Unclear & High & Unclear & Low & Low & Low \\
\hline Gronberg, 2009 & Low & High & Unclear & Low & Low & High \\
\hline Harada, 2018 & Unclear & High & Low & Low & Low & Unclear \\
\hline Helbekkmo, 2007 & Low & High & Low & Low & Low & Low \\
\hline Hellmann, 2019 & Low & High & Low & Low & Low & High \\
\hline Herbst, 2020 & Low & High & Low & Low & Low & High \\
\hline Kader, 2013 & High envelope & High & Low & Low & Low & Low \\
\hline Kaira, 2019 & Low & High & Low & Low & Low & High \\
\hline Kawahara, 2013 & Low & High & Low & Low & Low & Low \\
\hline Khodadad, 2014 & Unclear & High & Low & Low & Low & High \\
\hline Kubota, 2015 & Low & High & Low & Low & Low & High \\
\hline Langer, 2016 & Low & High & Unclear & Low & Low & High \\
\hline Lee, 2020 & Unclear & Low & Low & Unclear & Low & High \\
\hline Martoni, 2005 & Unclear & High & Unclear & Low & Low & Low \\
\hline Melosky, 2019 & Low & High & Low & Low & Low & High \\
\hline Minami, 2013 & Unclear & High & Low & Low & Low & Low \\
\hline Mok, 2019, NSq & Low & High & Low & Low & Low & High \\
\hline Niho, 2012 & Low & High & Low & Low & Low & High \\
\hline Novell, 2017 & Low & High & Low & Low & Low & High \\
\hline Ohe, 2007 & Low & High & Low & Low & Low & High \\
\hline Okamoto, 2010 & Low & High & Low & Low & Low & High \\
\hline $\begin{array}{l}\text { Papadimitrakopoulou, } \\
2018\end{array}$ & Unclear & High & Unclear & Unclear & Low & High \\
\hline Ouyang, 2018 & Low & Low & Low & Low & Low & High \\
\hline Patel, 2013 & Unclear & High & Low & Low & Low & High \\
\hline Paz-Ares, 2020 & Low & Low & Low & Low & Low & High \\
\hline $\begin{array}{l}\text { Ramalingam, 2017, } \\
\text { NSq }\end{array}$ & Low & Low & Low & Low & Low & High \\
\hline Reck, 2009 & Unclear & High & Low & Low & Low & High \\
\hline Rizvi, 2020 & Low & High & Low & Low & Low & High \\
\hline Rodrigues, 2011 & Low & High & Low & Low & Low & High \\
\hline Sandler, 2006 & Unclear & High & Low & Low & Low & Low \\
\hline Scagliotti, 2008 & Low & High & Low & Low & High & High \\
\hline Scagliotti, 2002 & Unclear & High & Low & Low & Low & High \\
\hline Schiller, 2002 & Unclear & High & Low & Low & Low & Low \\
\hline Smit, 2003 & Low & High & Low & Low & Low & High \\
\hline Socinski, 2018 & Low & High & Unclear & Low & Low & High \\
\hline Spigel, 2019 & Low & Low & Low & Low & Low & High \\
\hline Sun, 2015 & Unclear & High & Low & Low & Low & High \\
\hline Tan, 2009 & Low & High & Low & Low & Low & Low \\
\hline Thomas, 2006 & Unclear & High & Low & Low & Low & High \\
\hline Treat, 2010 & Unclear & High & Low & Low & Low & High \\
\hline Von Pawel, 2018 & Low & Low & Low & Low & Low & High \\
\hline West, 2019 & Low & High & Low & Low & Low & High \\
\hline Wheatley, 2019 & Low & High & Low & Low & Low & High \\
\hline $\mathrm{Wu}, 2020$ & Unclear & High & Low & Low & Low & High \\
\hline Wu, 2014 & Low & High & Low & Low & Low & High \\
\hline Yang, 2012 & Unclear & High & Low & High & Low & Low \\
\hline Yang, 2020 & Low & Low & Unclear & Low & Low & High \\
\hline Zhang, 2013 & Low & High & Low & Low & Low & High \\
\hline Zhou, 2015 & Low & Low & Low & Low & Low & Low \\
\hline Zhou, 2020 & Low & High & Unclear & Low & Low & High \\
\hline Zhu, 2018 & Low & High & Unclear & Low & High & Low \\
\hline Zinner, 2015 & Unclear & High & Low & Low & Low & High \\
\hline
\end{tabular}

One study had a high risk of selection bias due to randomization using an envelope method, 59 studies had a high risk of performance bias due to a non-blinded study design, 1 study had a high risk of attrition bias because $21 \%$ of the randomized patients did not receive the assigned regimen, and 3 studies had a high risk of reporting bias because the primary endpoint was not specified. Forty-six studies were marked as having a high risk of other bias for potential conflicts of interest because the studies were directly funded or advised by pharmaceutical companies. 


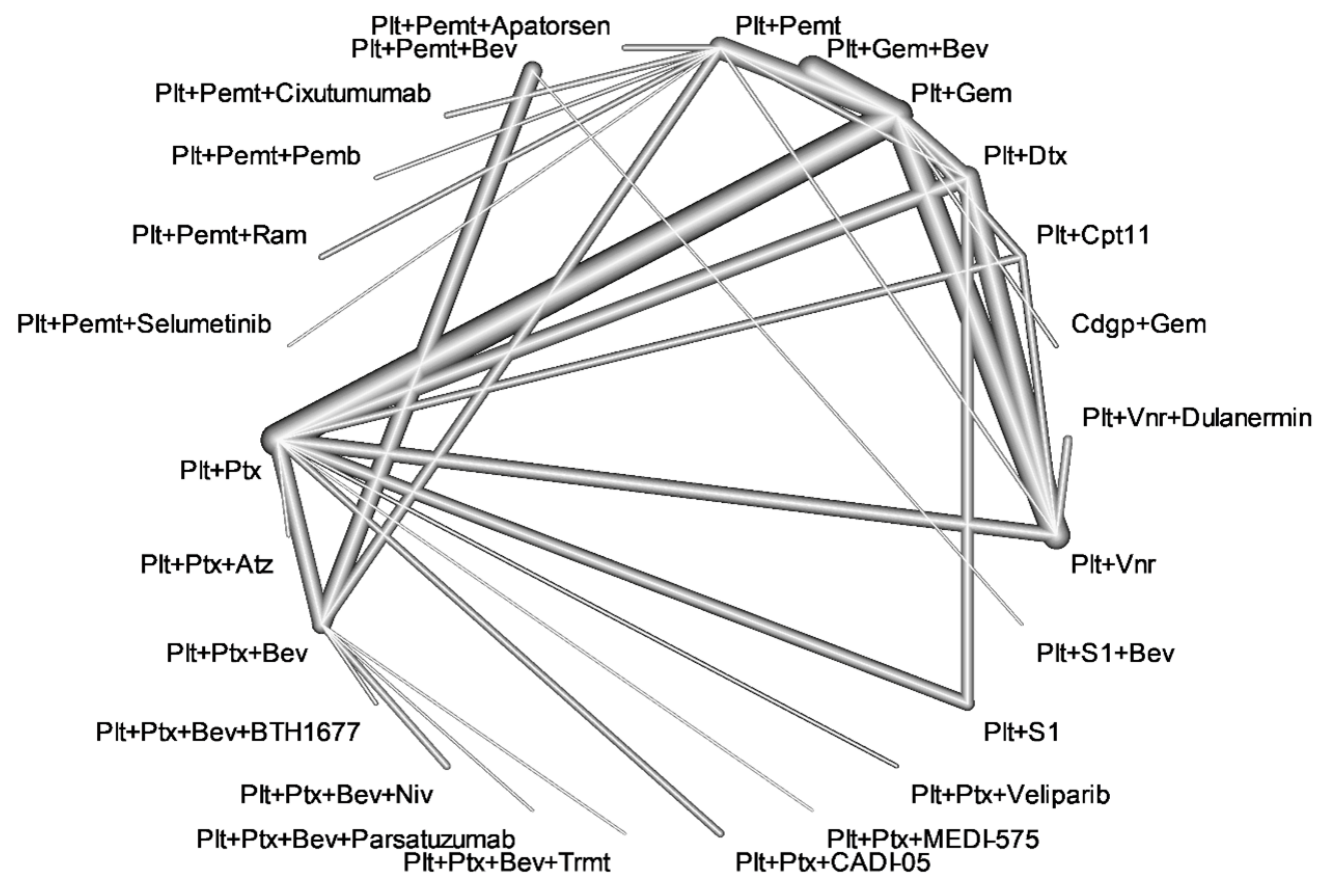

Figure S1 Network diagram for the primary endpoint, HR for OS. Separate model, Whole network level $\left(\mathrm{I}^{2}=0 \%, \mathrm{P}=0.8940\right.$, total; $\mathrm{P}=0.8940$, within designs; $\mathrm{P}=0.9491$, between designs; 0.4876). HR, hazard ratio; OS, overall survival; Plt, platinum regimen; Pemt, pemetrexed; Gem, gemcitabine; Dtx, docetaxel; Cpt11, irinotecan; Cdgp, nedaplatin; Vnr, vinorelbine; S1, tegafur gimeracil oteracil; Bev, bevacizumab; Ptx, paclitaxel; Trmt, tromethamine; Niv, nivolumab; Atz, atezolizumab; Ram, ramucirumab; Pemb, pembrolizumab.

\begin{tabular}{|c|c|c|c|c|}
\hline \multicolumn{5}{|c|}{ Comparison: other vs 'Plt_Regimen' } \\
\hline Treatment & (Random Ef & fects Mödel) & $\mathrm{HR}$ & $95 \%-\mathrm{Cl}$ \\
\hline Atz & & & 1.03 & {$[0.70 ; 1.52]$} \\
\hline Dur & & & 0.96 & {$[0.76 ; 1.22]$} \\
\hline Dur+Trml & & $\div$ & 1.16 & {$[0.92 ; 1.46]$} \\
\hline Niv & & & 1.32 & {$[0.71 ; 2.44]$} \\
\hline $\mathrm{Niv}+\mathrm{lpi}$ & & + & 1.03 & {$[0.75 ; 1.42]$} \\
\hline Pemb & $\longrightarrow$ & - & 0.83 & {$[0.62 ; 1.10]$} \\
\hline Plt Regimen & & & 1.00 & \\
\hline Plt Regimen+Apatorsen & & & 1.06 & {$[0.55 ; 2.04]$} \\
\hline Plt Regimen+Atz & 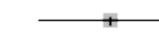 & & 0.70 & {$[0.45 ; 1.08]$} \\
\hline Plt_Regimen+BTH1677 & + & - & 0.75 & {$[0.44 ; 1.26]$} \\
\hline Plt Regimen+CADL-05 & $\longrightarrow$ & & 0.90 & {$[0.71: 1.15]$} \\
\hline Plt Regimen+Cixutumumab & $\longrightarrow$ & & 0.94 & {$[0.74: 120]$} \\
\hline Plt Regimen+Dulanermin & $\ldots$ & & 0.97 & {$[0.70 ; 1.34]$} \\
\hline Plt Regimen+MEDI-575 & & & 1.64 & {$[0.82 ; 3.28]$} \\
\hline Plt Regimen+Niv & 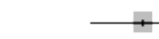 & - & 0.86 & {$[0.63 ; 1.18]$} \\
\hline Plt_Regimen+Niv+lpi & 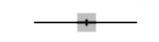 & & 0.61 & {$[0.44 ; 0.84]$} \\
\hline Plt_Regimen+Parsatuzumab & & + & 1.20 & {$[0.91 ; 1.58]$} \\
\hline Plt Regimen+Pemb & 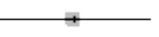 & & 0.55 & {$[0.34 ; 0.89]$} \\
\hline Plt_Regimen+Ram & & & 1.06 & {$[0.74 ; 1.52]$} \\
\hline Plt_Regimen+Trmt & & & 1.10 & {$[0.52 ; 2.31]$} \\
\hline Plt_Regimen+Veliparib & $\longrightarrow$ & & 0.90 & {$[0.51 ; 1.58]$} \\
\hline & 0.5 & 2 & & \\
\hline
\end{tabular}

Figure S2 Forest plots for primary outcomes (HR for OS) in main model that excepted conference abstracts. HR, hazard ratio; OS, overall survival; Plt, platinum regimen; Pemb, pembrolizumab; Niv, nivolumab; Ipi, ipilimumab; Dur, durvalumab; Trml, tremelimumab; Atz, atezolizumab; Trmt, tromethamine; Ram, ramucirumab; CI, confidence interval. 
A

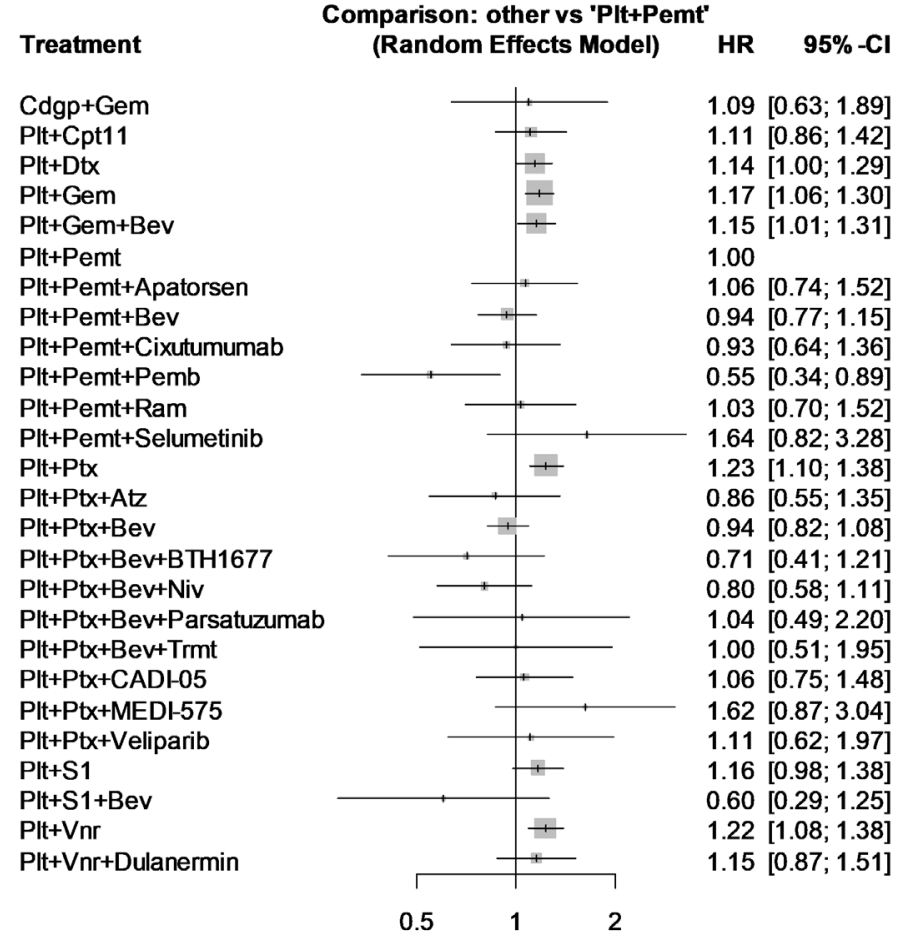

\section{C}

Treatment

Niv+lpi

Plt+Dtx

$\mathrm{Plt}+\mathrm{Gem}$

Plt+Pemt

Plt+Pemt+Atz

Plt+Pemt+Camrelizumab

Plt+Pemt+Cixutumumab

Plt+Pemt+Pemb

Plt+Pemt+Ram

Plt+Pemt+Sint

Plt+Ptx

Plt+Ptx+Atz

$\mathrm{Plt}+\mathrm{Ptx}+\mathrm{Bev}$

$\mathrm{Plt}+\mathrm{Ptx}+\mathrm{Bev}+\mathrm{Atz}$

Plt+Ptx+Bev+BTH1677

Plt+Ptx+Bev+Niv

Plt+Ptx+Bev+Parsatuzumab

Plt+Ptx+Bev+Trmt

Plt+Ptx+MEDI-575

Plt+Ptx+Veliparib

$\mathrm{Plt}+\mathrm{Vnr}$

Plt+Vnr+Dulanermin

Comparison: other vs 'PIt+Pemt'

(Random Effects Model) OR $\quad 95 \%$-Cl Treatment

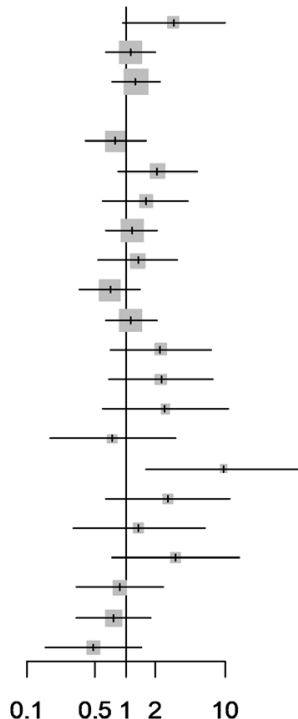

B

Treatment

Cdgp+Gem

Plt+Dtx

$\mathrm{Plt}+\mathrm{Gem}$

$\mathrm{Plt}+\mathrm{Gem}+\mathrm{Bev}$

Plt+Pemt

Plt+Pemt+Apatorsen

Plt+Pemt+Atz

$\mathrm{Plt}+\mathrm{Pemt}+\mathrm{Bev}$

Plt+Pemt+Camrelizumab

$\mathrm{Plt}+\mathrm{Pemt}+$ Cixutumumab

Plt+Pemt+Pemb

Plt+Pemt+Ram

Plt+Pemt+Selumetinib

Plt+Pemt+Sint

$P f t+P t x$

Plt+Ptx+Atz

$\mathrm{Plt}+\mathrm{Ptx}+\mathrm{Bev}$

$P l t+P t x+B e v+A t z$

$\mathrm{Plt}+\mathrm{Ptx}+\mathrm{Bev}+\mathrm{BTH} 1677$

$\mathrm{Plt}+\mathrm{Ptx}+\mathrm{Bev}+\mathrm{Niv}$

Plt+Ptx+Bev+Parsatuzumab

$\mathrm{Plt}+\mathrm{Ptx}+\mathrm{Bev}+\mathrm{Trmt}$

PIt+Ptx+CAD1-05

PIt+Ptx+MEDI-575

Plt+Ptx+Veliparib

$\mathrm{Plt}+\mathrm{S} 1$

$\mathrm{Plt}+\mathrm{S} 1+\mathrm{Bev}$

$\mathrm{Plt}+\mathrm{Vnr}$

$\mathrm{Plt}+\mathrm{Vnr}+$ Dulanermin
Comparison: other vs 'PIt+Pemt' (Random Effects Model) HR 95\% -C

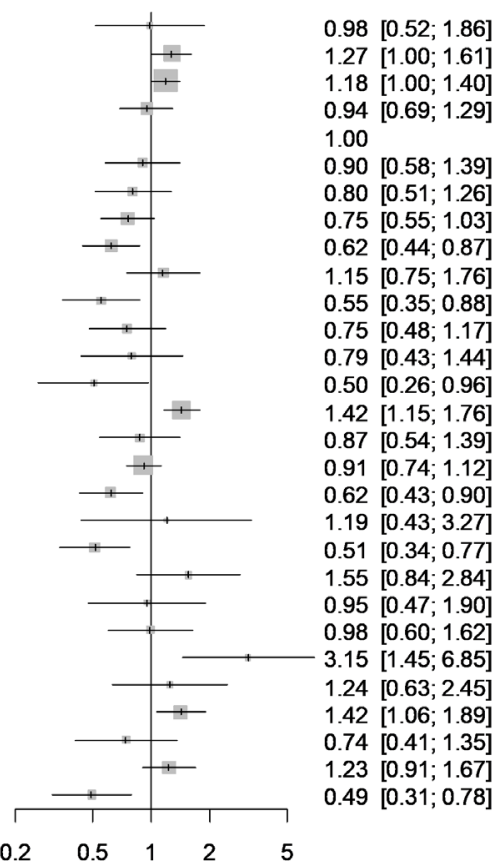

D

Comparison: other vs 'PIt+Pemt' (Random Effects Model) OR $\quad 95 \%-C$

$3.05[0.94 ; 9.88]$ Cdgp+Gem

$1.12[0.62 ; 2.02] \mathrm{Niv}+\mathrm{pi}$

1.27 [0.73; 2.21] Plt+Cpt11

PIt+Dtx

$0.80[0.40 ; 1.59] \mathrm{Plt}+\mathrm{Gem}$

$0.80[0.40 ; 1.59] \mathrm{Plt}+\mathrm{Gem}+\mathrm{Bev}$

$2.09[0.84 ; 5.20]$ Plt+Pemt

$1.60[0.59 ; 4.31]$ Plt+Pemt+Apatorsen

$1.16[0.64 ; 2.11]$ Plt+Pemt+At

$1.33[0.53 ; 3.36]$ Plt+Pemt+Bev

$0.70[0.35 ; 1.39]$ Plt+Pemt+Cixutumumab

$1.13[0.62 ; 2.06]$ Plt+Pemt+Pemb

$2.25[0.70 ; 7.25] \quad$ Plt+Pemt+Ram

$2.29[0.69 ; 7.62] \quad$ Plt+Pemt+Sint

$2.52[0.59 ; 10.76] \quad \mathrm{Plt}+\mathrm{Ptx}$

$0.74[0.17 ; 3.14] \quad \mathrm{Plt}+\mathrm{Ptx}+\mathrm{Atz}$

$9.71[1.61 ; 58.67] \quad P l t+P t x+B e v$

$2.66[0.63 ; 11.27] \mathrm{Plt}+\mathrm{Ptx}+\mathrm{Bev}+\mathrm{At}$

$1.36[0.30 ; 6.20] \quad \mathrm{Plt}+\mathrm{Ptx}+\mathrm{Bev}+\mathrm{BTH} 1677$

3.18 [0.73; 13.92] Plt+Ptx+Bev+Parsatuzumab

Plt+Ptx+CADI-05

$0.88[0.32 ; 2.40] \mathrm{Plt}+\mathrm{S} 1$

$0.76[0.33 ; 1.79]$ Plt+S1+Bev

$0.47[0.15 ; 1.46] \mathrm{Plt}+\mathrm{Vnr}$

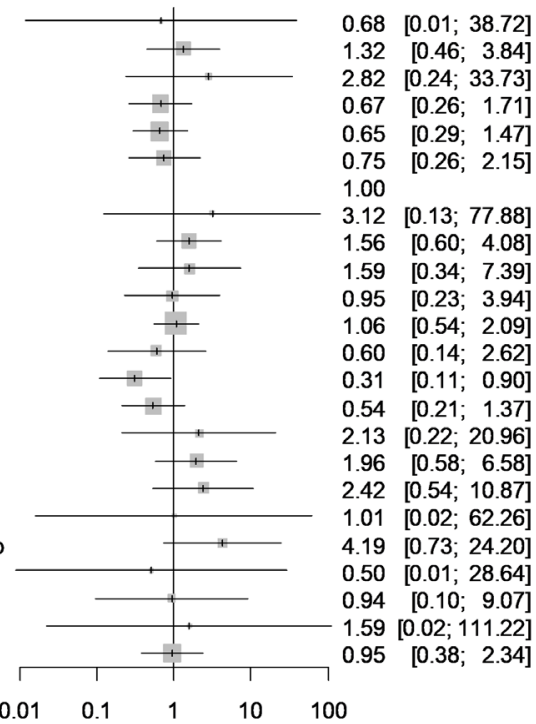

Figure S3 Forest plots for primary and secondary outcomes in separate model. (A) HR for OS. (B) HR for PFS. (C) OR for adverse events ( $\geq$ grade 3). (D) OR for chemo related death. HR, hazard ratio; OS, overall survival; PFS, progression-free survival; OR, odds ratio; Cdgp, nedaplatin; Gem, gemcitabine; Plt, platinum regimen; Cpt11, irinotecan; Dtx, docetaxel; Bev, bevacizumab; Pemt, pemetrexed; Pemb, pembrolizumab; Ram, ramucirumab; Ptx, paclitaxel; Atz, atezolizumab; Niv, nivolumab; Trmt, tromethamine; S1, tegafur gimeracil oteracil; Vnr, vinorelbine; Sint, sintilimab; CI, confidence interval. 